\title{
Embedded loops in the hyperbolic plane with prescribed, almost constant curvature
}

\author{
Roberta Musina* $\quad$ Fabio Zuddas ${ }^{\dagger}$
}

\begin{abstract}
Given a constant $k>1$ and a real valued function $K$ on the hyperbolic plane $\mathbb{H}^{2}$, we study the problem of finding, for any $\varepsilon \approx 0$, a closed and embedded curve $u^{\varepsilon}$ in $\mathbb{H}^{2}$ having geodesic curvature $k+\varepsilon K\left(u^{\varepsilon}\right)$ at each point.
\end{abstract}

\section{Introduction}

Let $\Sigma$ be an oriented Riemannian surface with empty boundary, Riemannian metric tensor $g$ and Levi-Civita connection $\nabla^{\Sigma}$. The geodesic curvature of a regular loop $u \in C^{2}\left(\mathbb{S}^{1}, \Sigma\right)$ is given by

$$
K(u)=\frac{\left\langle\nabla_{u^{\prime}}^{\Sigma} u^{\prime}, i_{u} u^{\prime}\right\rangle_{g}}{\left|u^{\prime}\right|_{g}^{3}} .
$$

Here we denoted by $i_{u}: T_{u} \Sigma \rightarrow T_{u} \Sigma$ the isometry that rotates $T_{u} \Sigma$, in such a way that $\left\{\tau, i_{u} \tau\right\}$ is a positively oriented orthogonal basis of $T_{u} \Sigma$, for any $\tau \neq 0$.

Given a sufficiently smooth function $K: \Sigma \rightarrow \mathbb{R}$, the $K$-loop problem consists in finding regular curves $u \in C^{2}\left(\mathbb{S}^{1}, \Sigma\right)$ having geodesic curvature $K(u)$ at each point. This problem can be faced by studying the system of ordinary differential equations

$$
\nabla_{u^{\prime}}^{\Sigma} u^{\prime}=L^{\Sigma}(u) K(u) i_{u} u^{\prime}, \quad L^{\Sigma}(u):=\left(f_{\mathbb{S}^{1}}\left|u^{\prime}\right|_{g}^{2} d x\right)^{\frac{1}{2}} .
$$

*Dipartimento di Scienze Matematiche, Informatiche e Fisiche, Università di Udine, via delle Scienze, 206 - 33100 Udine, Italy. Email: roberta.musina@uniud.it. Partially supported by Miur-PRIN project 2015KB9WPT_001.

${ }^{\dagger}$ Dipartimento di Matematica e Informatica, Università di Cagliari, Palazzo delle Scienze, Via Ospedale 72 - 09124 Cagliari, Italy. Email: fabio.zuddas@unica.it. 
Indeed, every nonconstant solution $u \in C^{2}\left(\mathbb{S}^{1}, \Sigma\right)$ to (1.1) has constant speed $\left|u^{\prime}\right|_{g}=$ $L^{\Sigma}(u)$, use for instance the computations in [14, Chapter 4]. Therefore $u$ is regular, and has curvature $K(u)$ at each point.

The $K$-loop problem has been largely studied since the seminal work [4] by Arnol'd. Most of the available existence results require compact target surfaces $\Sigma$; we limit ourselves to cite [9, 12, 13, 19, 20, 21, 22, 23] and references therein.

In the present paper we take $\Sigma$ to be the (noncompact) hyperbolic plane $\mathbb{H}^{2}$. It turns out that the problem under consideration does not have solutions, in general (see Subsection 2.2). In particular, if $-1 \leq K(q) \leq 1$ for any $q \in \Sigma$, then no $K$-loop exists. If $K \equiv k>1$ is constant (recall that changing the orientation of a curve changes the sign of its curvature), then any regular parameterization of an hyperbolic circle of radius

$$
\rho_{k}=\operatorname{artanh} \frac{1}{k}=\frac{1}{2} \ln \frac{k+1}{k-1}
$$

is a $k$-loop; conversely, any $k$-loop in $\mathbb{H}^{2}$ parameterizes some circle of radius $\rho_{k}$.

Our existence results involve curvatures that are small perturbations of a given constant $k>1$. In Section 3 we carefully choose a reference parameterization $\omega$ of a circle of radius $\rho_{k}$. Then we take any point $z \in \mathbb{H}^{2}$ and compose $\omega$ with an hyperbolic translation to obtain a parameterization $\omega_{z}$ of $\partial D_{\rho_{k}}^{\mathbb{H}}(z)$. Next, given $K \in C^{1}\left(\mathbb{H}^{2}\right)$, we look for a point $z_{0} \in \mathbb{H}^{2}$ and for embedded $(k+\varepsilon K)$-loops in $\mathbb{H}^{2}$ that suitably approach the circle $\omega_{z_{0}}$ as $\varepsilon \rightarrow 0$.

The center $z_{0}$ can not be arbitrarily prescribed. In fact, in Theorem 4.1 we prove that if there exists a sequence of $\left(k+\varepsilon_{h} K\right)$-loops $u_{h}$ such that $\varepsilon_{h} \rightarrow 0$ and $u_{h} \rightarrow \omega_{z_{0}}$ suitably, then $z_{0}$ is a critical point for the Melnikov-type function

$$
F_{k}^{K}(z)=\int_{D_{\rho_{k}}^{\mathbb{H}}(z)} K(z) d V_{\mathbb{H}}, \quad F_{k}^{K}: \mathbb{H}^{2} \rightarrow \mathbb{R} .
$$

One may wonder whether the existence of a critical point $z_{0}$ for $F_{k}^{K}$ is sufficient to have the existence, for $\varepsilon \approx 0$, of an embedded $(k+\varepsilon K)$-loop $u_{\varepsilon} \approx \omega_{z_{0}}$. We can give a positive answer in case $F_{k}^{K}$ has a stable critical point, accordingly with the next definition (see also [3, Chapter 2]).

Definition Let $X \in C^{1}\left(\mathbb{H}^{2}\right)$ and let $A \Subset \mathbb{H}^{2}$ be an open set. We say that $X$ has a stable critical point in $A$ if there exists $r>0$ such that any function $G \in C^{1}(\bar{A})$ satisfying $\|G-X\|_{C^{1}(\bar{A})}<r$ has a critical point in $A$. 
Sufficient conditions to have the existence of a stable critical point $z \in A$ for $X$ are easily given via elementary calculus. For instance, one can assume that one of the following conditions holds:

i) $\nabla X(z) \neq 0$ for any $z \in \partial A$, and $\operatorname{deg}(\nabla \mathrm{X}, \mathrm{A}, 0) \neq 0$, where "deg" is Browder's topological degree;

ii) $\min _{\partial A} X>\min _{A} X$ or $\max _{\partial A} X<\max _{A} X$

iii) $X$ is of class $C^{2}$ on $A$, it has a critical point $z_{0} \in A$, and the Hessian matrix of $X$ at $z_{0}$ is invertible.

We are in position to state our main result.

Theorem 1.1 Let $k>1$ and $K \in C^{1}\left(\mathbb{H}^{2}\right)$ be given. Assume that $F_{k}^{K}$ has a stable critical point in an open set $A \Subset \mathbb{H}^{2}$. Then for every $\varepsilon \in \mathbb{R}$ close enough to 0 , there exists an embedded $(k+\varepsilon K)$-loop $u^{\varepsilon}$.

Moreover, any sequence $\varepsilon_{h} \rightarrow 0$ has a subsequence $\varepsilon_{h_{j}}$ such that $u^{\varepsilon_{h_{j}}} \rightarrow \omega_{z_{0}}$ in $C^{2}\left(\mathbb{S}^{1}, \mathbb{H}^{2}\right)$ as $j \rightarrow \infty$, where $z_{0} \in \bar{A}$ is a critical point for $F_{k}^{K}$. In particular, if a point $z_{0} \in A$ is the unique critical point for $F_{k}^{K}$ in $\bar{A}$, then $u^{\varepsilon} \rightarrow \omega_{z_{0}}$ in $C^{2}\left(\mathbb{S}^{1}, \mathbb{H}^{2}\right)$ as $\varepsilon \rightarrow 0$.

Any stable critical point of the perturbation term $K$ gives rise to a stable critical point for $F_{k}^{K}$, at least for $k$ large enough. This is in essence the argument we use in Theorem 4.3 to obtain, via Theorem [1.1, the existence of $k+\varepsilon K$-loops whenever the perturbation curvature $K$ admits stable critical points.

The proof of Theorem 1.1 is based on a Lyapunov-Schmidt reduction technique combined with variational arguments, as proposed in [1] (see also [3, Chapter 2]).

In fact, $(k+\varepsilon K)$-loops correspond to critical points of an energy functional $E_{k+\varepsilon K}(u)=E_{k+\varepsilon K}(u)$, where $u$ runs in the class of nonconstant curves in $C^{2}\left(\mathbb{S}^{1}, \mathbb{H}^{2}\right)$ (see Section 2.1 for details). In particular, critical points of the unperturbed functional $E_{k}$ are circles of radius $\rho_{k}$. Let $\mathcal{S}=\left\{\omega_{z} \circ \xi\right\}$, where $\xi$ is a rotation of $\mathbb{S}^{1}$, $z \in \mathbb{H}^{2}$, and $\omega_{z}$ is our reference parameterization of $\partial D_{\rho_{k}}^{\mathbb{H}}(z)$. Clearly $\mathcal{S}$ is a smooth three-dimensional manifold of solutions to the unperturbed problem $E_{k}^{\prime}(u)=0$.

The crucial and technically difficult nondegeneracy result is proved in Lemma 3.3. via an efficient functional change inspired by [17]. It states that for any $z \in \mathbb{H}^{2}$, the tangent space to $\mathcal{S}$ at $\omega_{z}$ coincides with the set of solutions to the linear problem 
$E_{k}^{\prime \prime}\left(\omega_{z}\right) \varphi=0$. In the last section we carry out the dimensional reduction argument and complete the proof of Theorem 1.1.

We conclude the paper with a short appendix about the much more easy problem of finding loops in $\mathbb{R}^{2}$ having prescribed, almost constant curvature.

The Lyapunov-Schmidt reduction argument has been successfully used to study related geometrical problems. We limit ourselves to cite the pioneering paper [24] by R. Ye, [2, 6, 7, 8, 10, 11, 16, 17] and references therein.

\section{Notation and preliminaries}

The Euclidean space $\mathbb{R}^{2}$ is endowed with the scalar product $p \cdot q$ and norm $|\cdot|$, so that the disk of radius $R$ centered at $p \in \mathbb{R}^{2}$ is $D_{R}(p)=\left\{z \in \mathbb{R}^{2}|| z-p \mid<R\right\}$. The canonical basis of $\mathbb{R}^{2}$ is $e_{1}=(1,0), e_{2}=(0,1)$.

Let $A, \Omega \subseteq \mathbb{R}^{2}$ be open sets. We write $A \Subset \Omega$ if $\bar{A}$ is a compact subset of $\Omega$.

We will often use complex notation for points in $\mathbb{R}^{2}$. In particular we write $i z=\left(-z_{2}, z_{1}\right)$ and $z^{2}=\left(z_{1}^{2}-z_{2}^{2}, 2 z_{1} z_{2}\right)$ for $z=\left(z_{1}, z_{2}\right) \in \mathbb{R}^{2}$.

Let $\mathbb{S}^{1}$ be the unit circle in the complex plane. Any $\xi \in \mathbb{S}^{1}$ is identified with the rotation $x \mapsto \xi x$.

\section{The Poincaré half-plane model}

We adopt as model for the two dimensional hyperbolic space the half-plane

$$
\mathbb{H}^{2}=\left\{z=\left(z_{1}, z_{2}\right) \in \mathbb{R}^{2} \mid z_{2}>0\right\}
$$

endowed with the Riemannian metric $g_{l j}(z)=z_{2}^{-2} \delta_{l j}$. With some abuse of notation, we use the symbol $\mathbb{H}^{2}$ to denote the Euclidean upper half space as well.

The hyperbolic distance $d_{\mathbb{H}}(p, q)$ in $\mathbb{H}^{2}$ is related to the Euclidean one by

$$
\cosh d_{\mathbb{H}}(p, q)=1+\frac{|p-q|^{2}}{2 p_{2} q_{2}}
$$

and the hyperbolic disk $D_{\rho}^{\mathbb{H}}(p)$ centered at $p=\left(p_{1}, p_{2}\right)$ is the Euclidean disk of center $\left(p_{1}, p_{2} \cosh \rho\right)$ and radius $p_{2} \sinh \rho$.

A loop in the 2-dimensional hyperbolic space $\mathbb{H}^{2}$ is a curve $u: \mathbb{S}^{1} \rightarrow \mathbb{H}^{2}$ of class $C^{2}$ having nonzero derivative at each point. We say that $u$ is embedded if it is injective. 
If $G: \mathbb{H}^{2} \rightarrow \mathbb{R}$ is a differentiable function, then $\nabla^{\mathbb{H}} G(z)=z_{2}^{2} \nabla G(z)$, where $\nabla^{\mathbb{H}}, \nabla$ are the hyperbolic and the Euclidean gradients, respectively. In particular, $\nabla^{\mathbb{H}} G(z)=0$ if and only if $\nabla G(z)=0$.

The hyperbolic volume form $d V_{\mathbb{H}}$ is related to the Euclidean one by $d V_{\mathbb{H}}=z_{2}^{-2} d z$.

The Levi-Civita connection in $\mathbb{H}^{2}$ along a curve $u$ in $\Sigma$ is given by

$$
\nabla_{u^{\prime}}^{\mathbb{H}} u^{\prime}=u^{\prime \prime}-u_{2}^{-1} \Gamma\left(u^{\prime}\right),
$$

where, in complex notation, $\Gamma(z)=-i z^{2}$. In coordinates we have

$$
\Gamma(z):=\left(2 z_{1} z_{2}, z_{2}^{2}-z_{1}^{2}\right)=z_{2} z-z_{1} i z, \quad \Gamma: \mathbb{H}^{2} \rightarrow \mathbb{R}^{2}
$$

For future convenience we compute the differential

$$
\Gamma^{\prime}(z) w=2\left(w_{2} z-w_{1} i z\right), \quad z \in \mathbb{H}^{2}, w \in \mathbb{R}^{2}
$$

Isometries in $\mathbb{H}^{2}$

Hyperbolic translations are obtained by composing a horizontal (Euclidean) translation $w \mapsto w+s e_{1}, s \in \mathbb{R}$ (sometimes called parabolic isometry), with an Euclidean homothety $w \mapsto t w, t>0$ (in some literature, only homotheties are called hyperbolic translations). We obtain the two dimensional group of isometries $\mathbb{H}^{2} \rightarrow \mathbb{H}^{2}$,

$$
u \mapsto u_{z}:=z_{1} e_{1}+z_{2} u, \quad z \in \mathbb{H}^{2}
$$

\section{Function spaces}

Let $m \geq 0, n \geq 1$ be integer numbers. We endow $C^{m}\left(\mathbb{S}^{1}, \mathbb{R}^{n}\right)$ with the standard Banach space structure. If $f \in C^{1}\left(\mathbb{S}^{1}, \mathbb{R}^{n}\right)$, we identify $f^{\prime}(x) \equiv f^{\prime}(x)(i x)$, so that $f^{\prime}: \mathbb{S}^{1} \rightarrow \mathbb{R}^{n}$.

In $L^{2}=L^{2}\left(\mathbb{S}^{1}, \mathbb{R}^{2}\right)$ we take the Hilbertian norm

$$
\|u\|_{L^{2}}^{2}=f_{\mathbb{S}^{1}}|u(x)|^{2} d x=\frac{1}{2 \pi} \int_{\mathbb{S}^{1}}|u(x)|^{2} d x .
$$

If $T \subseteq C^{0}\left(\mathbb{S}^{1}, \mathbb{R}^{2}\right)$, the orthogonal to $T$ with respect to the $L^{2}$ scalar product is

$$
T^{\perp}=\left\{\varphi \in C^{0}\left(\mathbb{S}^{1}, \mathbb{R}^{2}\right) \mid f_{\mathbb{S}^{1}} u \cdot \varphi d x=0 \text { for any } u \in T\right\} .
$$

We look at $C^{m}\left(\mathbb{S}^{1}, \mathbb{H}^{2}\right)$ as an open subset of the Banach space $C^{m}\left(\mathbb{S}^{1}, \mathbb{R}^{2}\right)$, and

identify $\mathbb{H}^{2}$ with the set of constant functions in $C^{m}\left(\mathbb{S}^{1}, \mathbb{H}^{2}\right)$. Thus $C^{m}\left(\mathbb{S}^{1}, \mathbb{H}^{2}\right) \backslash \mathbb{H}^{2}$ contains only nonconstant curves. 


\subsection{The variational approach}

We put

$$
L(u):=L_{\mathbb{H}^{2}}(u)=\left(f_{\mathbb{S}^{1}} u_{2}^{-2}\left|u^{\prime}\right|^{2} d x\right)^{\frac{1}{2}}, \quad L: C^{2}\left(\mathbb{S}^{1}, \mathbb{H}^{2}\right) \rightarrow \mathbb{R},
$$

that is a $C^{\infty}$ functional, with Fréchet differential

$$
L^{\prime}(u) \varphi=\frac{1}{L(u)} f_{\mathbb{S}^{1}} u_{2}^{-2}\left(-u^{\prime \prime}+u_{2}^{-1} \Gamma\left(u^{\prime}\right)\right) \cdot \varphi d x, \quad \varphi \in C^{2}\left(\mathbb{S}^{1}, \mathbb{R}^{2}\right) .
$$

When $\Sigma=\mathbb{H}^{2}$, problem (1.1) reads

$$
u^{\prime \prime}-u_{2}^{-1} \Gamma\left(u^{\prime}\right)=L(u) K(u) i u^{\prime} .
$$

The system $\left(\mathcal{P}_{K}\right.$ admits a variational formulation. More precisely, its nonconstant solutions are critical points of the energy functional of the form

$$
E_{K}(u)=L(u)+A_{K}(u), \quad u \in C^{2}\left(\mathbb{S}^{1}, \mathbb{H}^{2}\right) \backslash \mathbb{H}^{2},
$$

where $A_{K}(u)$ gives, roughly speaking, the signed area enclosed by the curve $u$ with respect to the weight $K$ (see Remark 2.2 below). More precisely, to introduce $A_{K}(u)$ we take any vectorfield $Q_{K} \in C^{1}\left(\mathbb{H}^{2}, \mathbb{R}^{2}\right)$ such that

$$
\operatorname{div} Q_{K}(z)=z_{2}^{-2} K(z), \quad z \in \mathbb{H}^{2}
$$

(here "div" is the usual Euclidean divergence). A possible choice is

$$
Q_{K}\left(z_{1}, z_{2}\right)=\left(\frac{1}{2} z_{2}^{-2} \int_{0}^{z_{1}} K\left(t, z_{2}\right) d t\right) e_{1}+\left(\frac{1}{2} \int_{1}^{z_{2}} t^{-2} K\left(z_{1}, t\right) d t\right) e_{2} .
$$

Then we define

$$
A_{K}(u)=f_{\mathbb{S}^{1}} Q_{K}(u) \cdot i u^{\prime} d x, \quad A_{K}: C^{2}\left(\mathbb{S}^{1}, \mathbb{H}^{2}\right) \rightarrow \mathbb{R} .
$$

By direct computations one gets that the functional $A_{K}$ is Fréchet differentiable at any $u \in C^{2}\left(\mathbb{S}^{1}, \mathbb{H}^{2}\right)$, with differential

$$
A_{K}^{\prime}(u) \varphi=f_{\mathbb{S}^{1}} u_{2}^{-2} K(u) \varphi \cdot i u^{\prime} d x . \quad \varphi \in C^{2}\left(\mathbb{S}^{1}, \mathbb{R}^{2}\right),
$$

It follows that $A_{K}(u)$ does not depend on the choice of the vectorfield $Q_{K}$. Further, if $K \in C^{1}\left(\mathbb{H}^{2}\right)$ then the area functional $A_{K}$ is of class $C^{2}$ on $C^{2}\left(\mathbb{S}^{1}, \mathbb{R}^{2}\right)$.

In conclusion, the following lemma holds. 
Lemma 2.1 Let $K \in C^{1}\left(\mathbb{H}^{2}\right)$. The functional $E_{K}(u)=L(u)+A_{K}(u)$ is of class $C^{2}$ on $C^{2}\left(\mathbb{S}^{1}, \mathbb{H}^{2}\right) \backslash \mathbb{H}^{2}$, and

$$
L(u) E_{K}^{\prime}(u) \varphi=f_{\mathbb{S}^{1}} u_{2}^{-2}\left(-u^{\prime \prime}+u_{2}^{-1} \Gamma\left(u^{\prime}\right)+L(u) K(u) i u^{\prime}\right) \cdot \varphi d x
$$

for any $u \in C^{2}\left(\mathbb{S}^{1}, \mathbb{H}^{2}\right) \backslash \mathbb{H}^{2}, \varphi \in C^{2}\left(\mathbb{S}^{1}, \mathbb{R}^{2}\right)$. In particular, if $u_{0} \in C^{2}\left(\mathbb{S}^{1}, \mathbb{H}^{2}\right) \backslash \mathbb{H}^{2}$ is a critical point for the functional $E_{K}(u)$, then $u_{0}$ solves $\left(\overline{\mathcal{P}_{K}}\right)$, hence it is an hyperbolic $K$-loop.

Remark 2.2 Let $u \in C^{2}\left(\mathbb{S}^{1}, \mathbb{H}^{2}\right)$ be an embedded loop. Then $u$ is a regular parameterization of the boundary of an open set $\Omega_{u} \Subset \mathbb{H}^{2}$. Assume for instance that $u$ is positively oriented, so that iu gives the inner direction to $\Omega_{u}$. Then

$$
A_{K}(u)=-\frac{1}{2 \pi} \int_{\partial \Omega} Q_{K}(z) \cdot \nu d s=-\frac{1}{2 \pi} \int_{\Omega} K(z) d V_{\mathbb{H}}
$$

by the divergence theorem.

\subsection{Nonexistence results}

We start with a simple result that should be well known. We sketch its proof by adapting the argument in [15, p. 194].

Proposition 2.3 Let $K \in C^{0}\left(\mathbb{H}^{2}\right)$. If $\|K\|_{\infty} \leq 1$ then no $K$-loop exists.

Proof. Let $u \in C^{2}\left(\mathbb{S}^{1}, \mathbb{H}^{2}\right)$ be a $K$-loop. We need to show that $|K|>1$ somewhere in $\mathbb{H}^{2}$. Take the smallest closed disk $D_{\rho}=\overline{D_{\rho}^{\mathbb{H}}(z)}$ containing $u\left(\mathbb{S}^{1}\right)$. Then $\partial D_{\rho}$ is tangent to $u\left(\mathbb{S}^{1}\right)$ at some point. At the contact point the absolute value of the curvature of $u$ can not be smaller than the curvature $1 / \tanh \rho$ of the circle $\partial D_{\rho}$, use a local comparison principle. The conclusion readily follows from $\tanh \rho<1$.

Next, we point out few necessary conditions for the existence of $K$-loops.

Lemma 2.4 Let $K \in C^{1}\left(\mathbb{H}^{2}\right)$ and let $\Omega \subset \mathbb{H}^{2}$ be a bounded open domain. Assume that $\partial \Omega$ is parameterized by a $K$-loop $u \in C^{2}\left(\mathbb{S}^{1}, \mathbb{H}^{2}\right)$. Then

$$
\int_{\Omega} \nabla K(z) \cdot e_{1} d V_{\mathbb{H}^{2}}=\int_{\Omega} \nabla K(z) \cdot z d V_{\mathbb{H}^{2}}=\int_{\Omega} \nabla K(z) \cdot z^{2} d V_{\mathbb{H}^{2}}=0 .
$$


Proof. Direct computations based on integration by parts give

$$
L^{\prime}(u) e_{1}=L^{\prime}(u) u=L^{\prime}(u) i(\Gamma u)=0,
$$

see (2.4) and (2.2). In addition, the curve $u$ solves

$$
-L(u) L^{\prime}(u) \varphi=f_{\mathbb{S}^{1}} u_{2}^{-2} K(u) \varphi \cdot i u^{\prime} d x \quad \text { for any } \varphi \in C^{2}\left(\mathbb{S}^{1}, \mathbb{R}^{2}\right) .
$$

Since $i u^{\prime}(x) \neq 0$ is parallel to the outer normal $\nu$ to $\Omega$ at $u(x) \in \partial \Omega$, we infer that

$$
\int_{\partial \Omega} z_{2}^{-2} K(z) e_{1} \cdot \nu=\int_{\partial \Omega} z_{2}^{-2} K(z) z \cdot \nu=\int_{\partial \Omega} z_{2}^{-2} K(z) i \Gamma(z) \cdot \nu=0 .
$$

Recall that we identify $i \Gamma(z)=z^{2}$, then use the divergence theorem to get

$$
\int_{\Omega} \operatorname{div}\left(z_{2}^{-2} K(z) e_{1}\right) d z=\int_{\Omega} \operatorname{div}\left(z_{2}^{-2} K(z) z\right) d z=\int_{\Omega} \operatorname{div}\left(z_{2}^{-2} K(z) z^{2}\right) d z=0 .
$$

The conclusion readily follows.

Remark 2.5 The identities in (2.6) hold indeed for any curve $u$, and are related to the group of isometries in $\mathbb{H}^{2}$. Notice indeed that $z \mapsto e_{1}, z \mapsto z, z \mapsto z^{2}$ are infinitesimal Killing vectorfields in $\mathbb{H}^{2}$.

Lemma 2.4 readily implies the next nonexistence result.

Corollary 2.6 Let $K \in C^{1}\left(\mathbb{H}^{2}\right)$ be a given curvature function. Assume that one of the following conditions hold,

i) $K$ is strictly monotone in the $e_{1}$ direction;

ii) $K$ is radially strictly monotone, that is, $\nabla K(z) \cdot z$ never vanishes on $\mathbb{H}^{2}$;

iii) $\nabla K(z) \cdot z^{2}$ never vanishes on $\mathbb{H}^{2}$

Then no embedded $K$-loop exists. 


\section{The unperturbed problem}

In this section we take a constant $k>1$ and study the system

$$
u^{\prime \prime}-u_{2}^{-1} \Gamma\left(u^{\prime}\right)=L(u) k i u^{\prime} .
$$

We start by introducing the radius

$$
R_{k}:=\sinh \rho_{k}=\frac{1}{k} \cosh \rho_{k}=\frac{1}{\sqrt{k^{2}-1}}
$$

and the reference loop $\omega: \mathbb{S}^{1} \rightarrow \mathbb{H}^{2}$

$$
\omega(x)=\frac{1}{k-x_{2}}\left(x_{1}, \frac{1}{R_{k}}\right), \quad x=x_{1}+i x_{2} \in \mathbb{S}^{1} .
$$

Notice that

$$
\left|\omega-k R_{k} e_{2}\right|=R_{k}
$$

hence $\omega$ is a (positive) parametrization of the Euclidean circle $\partial D_{R_{k}}\left(k R_{k} e_{2}\right)$, that coincides with the hyperbolic circle $\partial D_{\rho_{k}}^{\mathbb{H}}\left(e_{2}\right)$. The next identities will be very useful:

$$
\begin{aligned}
& \omega^{\prime}=\omega_{2} i\left(\omega-k R_{k} e_{2}\right) \\
& \omega_{2}^{-1} \Gamma\left(\omega^{\prime}\right)=\left(\omega_{2}-k R_{k}\right) i \omega^{\prime}+\omega_{1} \omega^{\prime} \\
& \omega_{2}^{-1}\left|\omega^{\prime}\right| \equiv L(\omega)=R_{k} .
\end{aligned}
$$

By differentiating (3.3) and using (3.5) one easily gets that $\omega$ solves $\left(\mathcal{P}_{k}\right)$. Next, for $z=\left(z_{1}, z_{2}\right) \in \mathbb{H}^{2}$ we parameterize $\partial D_{\rho_{k}}^{\mathbb{H}}(z)$ by the function

$$
\omega_{z}=z_{1} e_{1}+z_{2} \omega
$$

Notice that $\omega=\omega_{e_{2}}$. It is easy to check that for any rotation $\xi \in \mathbb{S}^{1}$ and any point $z \in \mathbb{H}^{2}$, the circle $\omega_{z} \circ \xi$ solves $\left(\mathcal{P}_{k}\right.$ as well. Further, by Remark 2.2 we have

$$
F_{k}^{K}(z):=\int_{D_{\rho_{k}}^{\mathbb{H I}}(z)} K(z) d V_{\mathbb{H}}=-2 \pi A_{K}\left(\omega_{z}\right) .
$$

We know that any nonconstant solution $u$ to $\left(\mathcal{P}_{k}\right)$ has constant curvature $k$, hence is a circle of hyperbolic radius $\rho_{k}$. Actually we need a sharper uniqueness result, that is, we have to classify solutions to $\left(\mathcal{P}_{k}\right)$.

Lemma 3.1 Let $u \in C^{2}\left(\mathbb{S}^{1}, \mathbb{H}^{2}\right)$ be a nonconstant solution to $\overline{\mathcal{P}_{k}}$. Then $\mu:=L(u) / L(\omega)$ is an integer number, and there exist $\xi \in \mathbb{S}^{1}, z=\left(z_{1}, z_{2}\right) \in \mathbb{H}^{2}$ such that $u(x)=\omega_{z} \circ \xi$. In particular, $u$ parameterizes $\partial D_{\rho_{k}}(z)$, and $L(u)=\mu L(\omega)=\mu R_{k}$. 
Proof. We have

$$
\omega_{2}(-i)=e^{-\rho_{k}}=\min _{x \in \mathbb{S}^{1}} \omega_{2}(x), \quad \omega(-i)=e^{-\rho_{k}} e_{2}, \quad \omega^{\prime}(-i)=e^{-\rho_{k}} L(\omega) e_{1} .
$$

Let $x_{u} \in \mathbb{S}^{1}$ such that

$$
u_{2}\left(x_{u}\right)=m_{u}:=\min _{x \in \mathbb{S}^{1}} u_{2}(x)
$$

Now we show that

$$
u^{\prime}\left(x_{u}\right)=m_{u} L(u) e_{1} .
$$

Clearly $u_{2}^{\prime}\left(x_{u}\right)=0$ and $u_{2}^{\prime \prime}\left(x_{u}\right) \geq 0$. We first infer that $\Gamma\left(u^{\prime}\left(x_{u}\right)\right)=-u_{1}^{\prime}\left(x_{u}\right) i u^{\prime}\left(x_{u}\right)$, compare with (2.2). Thus the system $\left(\mathcal{P}_{k}\right)$ for the second coordinate gives

$$
\left(L(u) k-m_{u}^{-1} u_{1}^{\prime}\left(x_{u}\right)\right) u_{1}^{\prime}\left(x_{u}\right)=u_{2}^{\prime \prime}\left(x_{u}\right) \geq 0,
$$

that implies $u_{1}^{\prime}\left(x_{u}\right) \geq 0$. On the other hand, $u_{2}^{-1}\left|u^{\prime}\right| \equiv L(u)$ on $\mathbb{S}^{1}$. Thus $u_{1}^{\prime}\left(x_{u}\right)=$ $\left|u^{\prime}\left(x_{u}\right)\right|=m_{u} L(u)$, and (3.7) is proved.

In particular, $u$ solves the Cauchy problem

$$
v^{\prime \prime}=v_{2}^{-1} \Gamma\left(v^{\prime}\right)+k L(u) i v^{\prime}, \quad v\left(x_{u}\right)=u\left(x_{u}\right), \quad v^{\prime}\left(x_{u}\right)=m_{u} L(u) e_{1}
$$

It is easy to check that the function

$$
\tilde{u}(x):=m_{u} e^{\rho_{k}} \omega\left(-i x_{u}^{-\mu} x^{\mu}\right)+u_{1}\left(x_{u}\right) e_{1}
$$

solves (3.8) as well (use $f^{\prime}(x)=i \mu x^{\mu}$ for $f(x)=x^{\mu}, f: \mathbb{S}^{1} \rightarrow \mathbb{C}$ ). Thus $\tilde{u}(x)=u(x)$ for any $x \in \mathbb{S}^{1}$ and hence $u(x)=\omega_{z} \circ \xi$, where $z_{1}=u_{1}\left(x_{u}\right), z_{2}=m_{u} e^{\rho_{k}}, \xi=-i x_{u}^{-\mu}$. Finally, $\mu$ is an integer number because $u$ and $\omega$ are both well defined on $\mathbb{S}^{1}$.

\section{The linearized problem}

By Lemma 3.1, the 3-dimensional manifold

$$
\mathcal{S}=\left\{\omega_{z} \circ \xi \mid \xi \in \mathbb{S}^{1}, \quad z \in \mathbb{H}^{2}\right\} \subset C^{2}\left(\mathbb{S}^{1}, \mathbb{H}^{2}\right), \quad \omega_{z}=z_{1} e_{1}+z_{2} \omega
$$

is the set of embedded solutions to $\left(\mathcal{P}_{k}\right)$. The tangent space to $\mathcal{S}$ at $\omega_{z}$ is

$$
T_{\omega_{z}} \mathcal{S}=T_{\omega} \mathcal{S}=\left\langle\omega^{\prime}, e_{1}, \omega\right\rangle
$$

Every loop in $\omega_{z} \circ \xi \in \mathcal{S}$ is a critical point for the energy functional

$$
E_{k}(u)=L(u)+A_{k}(u)=\left(f_{\mathbb{S}^{1}} u_{2}^{-2}\left|u^{\prime}\right|^{2} d x\right)^{\frac{1}{2}}-k f_{\mathbb{S}^{1}} u_{2}^{-1} u_{1}^{\prime} d x
$$

on $C^{2}\left(\mathbb{S}^{1}, \mathbb{H}^{2}\right) \backslash H^{2}$, and $E_{k}\left(\omega_{z} \circ \xi\right)=E_{k}(\omega)$ is a constant. More generally one has

$$
E_{k}\left(z_{1} e_{1}+z_{2} u \circ \xi\right)=E_{k}(u) \quad \text { for any } \xi \in \mathbb{S}^{1}, z \in \mathbb{H}^{2} .
$$


In order to handle the differential of $E_{k}$, it is convenient to introduce the function $J_{0}: C^{2}\left(\mathbb{S}^{1}, \mathbb{H}^{2}\right) \backslash \mathbb{H}^{2} \rightarrow C^{0}\left(\mathbb{S}^{1}, \mathbb{H}^{2}\right)$ given by

$$
\begin{aligned}
J_{0}(u) & =-\left(u_{2}^{-2} u^{\prime}\right)^{\prime}-u_{2}^{-3}\left|u^{\prime}\right|^{2} e_{2}+L(u) k u_{2}^{-2} i u^{\prime} \\
& =u_{2}^{-2}\left(-u^{\prime \prime}+u_{2}^{-1} \Gamma\left(u^{\prime}\right)+L(u) k i u^{\prime}\right) .
\end{aligned}
$$

By Lemma 2.1 we have

$$
L(u) E_{k}^{\prime}(u) \varphi=f_{\mathbb{S}^{1}} J_{0}(u) \cdot \varphi d x \quad \text { for any } \varphi \in C^{2}\left(\mathbb{S}^{1}, \mathbb{R}^{2}\right) .
$$

By differentiating (3.9) at $\xi=1, z=e_{2}$ we readily get $E_{k}^{\prime}(u) u^{\prime}=E_{k}^{\prime}(u) e_{1}=E_{k}^{\prime}(u) u=0$ for any nonconstant curve $u \in C^{2}\left(\mathbb{S}^{1}, \mathbb{H}^{2}\right)$, that is,

$$
f_{\mathbb{S}^{1}} J_{0}(u) \cdot u^{\prime} d x=0, f_{\mathbb{S}^{1}} J_{0}(u) \cdot e_{1} d x=0, \quad f_{\mathbb{S}^{1}} J_{0}(u) \cdot u d x=0 .
$$

Now we differentiate (3.11) with respect to $u$, at $u=\omega_{z}$. From $E_{k}^{\prime}\left(\omega_{z}\right)=0$ we get

$$
L(\omega) E_{k}^{\prime \prime}\left(\omega_{z}\right)[\varphi, \tilde{\varphi}]=\int_{\mathbb{S}^{1}} J_{0}^{\prime}\left(\omega_{z}\right) \varphi \cdot \tilde{\varphi} d x \quad \text { for any } \varphi, \tilde{\varphi} \in C^{2}\left(\mathbb{S}^{1}, \mathbb{R}^{2}\right) .
$$

Since $E_{k}$ is of class $C^{2}$, then $J_{0}^{\prime}\left(\omega_{z}\right)$ is self-adjoint in $L^{2}$, that means

$$
f_{\mathbb{S}^{1}} J_{0}^{\prime}\left(\omega_{z}\right) \varphi \cdot \tilde{\varphi} d x=f_{\mathbb{S}^{1}} J_{0}^{\prime}\left(\omega_{z}\right) \tilde{\varphi} \cdot \varphi d x \quad \text { for any } \varphi, \tilde{\varphi} \in C^{2}\left(\mathbb{S}^{1}, \mathbb{R}^{2}\right) .
$$

Finally, we differentiate $E_{k}^{\prime}\left(\omega_{z} \circ \xi\right)=0$ with respect to the variables $\xi \in \mathbb{S}^{1}, z \in \mathbb{H}^{2}$ to get $T_{\omega_{z}} \mathcal{S} \subseteq \operatorname{ker} J_{0}^{\prime}\left(\omega_{z}\right)$. We shall see in the crucial Lemma 3.3 below that indeed $T_{\omega_{z}} \mathcal{S}=\operatorname{ker} J_{0}^{\prime}\left(\omega_{z}\right)$.

This will be done via a useful functional change.

\section{A functional change and nondegeneracy}

In order to avoid tricky computations, we use in $C^{m}\left(\mathbb{S}^{1}, \mathbb{R}^{2}\right), m \geq 0$, the orthogonal frame $\omega^{\prime}, i \omega^{\prime}$. We introduce the isomorphism

$$
\Phi(g)=g_{1} \omega^{\prime}+g_{2} i \omega^{\prime}, \quad \Phi: C^{m}\left(\mathbb{S}^{1}, \mathbb{R}^{2}\right) \rightarrow C^{m}\left(\mathbb{S}^{1}, \mathbb{R}^{2}\right)
$$

together with its inverse $\Phi^{-1}(\varphi)=R_{k}^{-2} \omega_{2}^{-2}\left(\varphi \cdot \omega^{\prime} e_{1}+\varphi \cdot i \omega^{\prime} e_{2}\right)$ (recall that $\left.\left|\omega^{\prime}\right|=R_{k} \omega_{2}\right)$ and the differential operator

$$
B g=-g^{\prime \prime}-k R_{k} i g^{\prime}+R_{k}^{2}\left(g_{2}-k^{2} f_{\mathbb{S}^{1}} g_{2} d x\right) e_{2}, \quad g \in C^{2}\left(\mathbb{S}^{1}, \mathbb{R}^{2}\right) .
$$


Lemma 3.2 Let $z$ be any point in $\mathbb{H}^{2}$. The following facts hold.

i) $J_{0}^{\prime}\left(\omega_{z}\right)(\Phi(g))=z_{2}^{-2} \omega_{2}^{-2} \Phi(B g)$ for any $g \in C^{2}\left(\mathbb{S}^{1}, \mathbb{S}^{2}\right)$;

ii) $f_{\mathbb{S}^{1}} \omega_{2}^{-2} \Phi(g) \cdot \Phi(\tilde{g}) d x=R_{k}^{2} f_{\mathbb{S}^{1}} g \cdot \tilde{g} d x$ for any $g, \tilde{g} \in C^{2}\left(\mathbb{S}^{1}, \mathbb{S}^{2}\right)$

Proof. Since $J_{0}\left(\omega_{z}\right)=z_{2}^{-1} J_{0}(\omega)=0$ and $J_{0}^{\prime}\left(\omega_{z}\right)=z_{2}^{-2} J_{0}^{\prime}(\omega)$, it suffices to prove $i$ ) for $z=e_{2}$, that corresponds to $\omega_{z}=\omega$. We have to show that

$$
\mathcal{J}(\varphi):=\omega_{2}^{2} J_{0}^{\prime}(\omega) \varphi=\Phi(B g), \quad \text { where } \varphi=g_{1} \omega^{\prime}+g_{2} i \omega^{\prime}
$$

To compute $\mathcal{J}(\varphi)$ it is convenient to recall (3.10) and to differentiate the identity

$$
u_{2}^{2} J_{0}(u)=-u^{\prime \prime}+u_{2}^{-1} \Gamma\left(u^{\prime}\right)+L(u) k i u^{\prime}
$$

at $u=\omega$. Since $J_{0}(\omega)=0$ and $L(\omega)=R_{k}$, we get

$$
\mathcal{J}(\varphi)=-\varphi^{\prime \prime}+k R_{k} i \varphi^{\prime}+\omega_{2}^{-1} \Gamma^{\prime}\left(\omega^{\prime}\right) \varphi^{\prime}-\omega_{2}^{-2} \varphi_{2} \Gamma\left(\omega^{\prime}\right)+k\left(L^{\prime}(\omega) \varphi\right) i \omega^{\prime} .
$$

From (2.3) we find $\Gamma^{\prime}\left(\omega^{\prime}\right) \varphi^{\prime}=2 \varphi_{2}^{\prime} \omega^{\prime}-2 \varphi_{1}^{\prime} i \omega^{\prime}$. Taking also (3.4) into account, we obtain

$$
\mathcal{J}(\varphi)=-\varphi^{\prime \prime}+k R_{k} i \varphi^{\prime}+A_{1}(\varphi) \omega^{\prime}-\left(A_{2}(\varphi)-k L^{\prime}(\omega) \varphi\right) i \omega^{\prime},
$$

where

$$
A_{1}(\varphi)=\left(2 \varphi_{2}^{\prime}-\varphi_{2} \omega_{1}\right) \omega_{2}^{-1}, \quad A_{2}(\varphi)=\left(2 \varphi_{1}^{\prime}+\varphi_{2}\left(\omega_{2}-k R_{k}\right)\right) \omega_{2}^{-1} .
$$

To compute the differential $L^{\prime}(\omega)$ at $\varphi$ we recall that $\omega$ solves $\left(\mathcal{P}_{k}\right)$. Thus (2.4) gives

$$
L^{\prime}(\omega) \varphi=-k f_{\mathbb{S}^{1}} \omega_{2}^{-2} \varphi \cdot i \omega^{\prime} d x .
$$

For the next computations we observe that the loop $\omega$ solves several useful differential systems. In particular, from $\left(\underline{\left.\mathcal{P}_{k}\right)},(3.4),(3.5)\right.$ and $(3.3)$ it follows that

$$
\omega^{\prime \prime}=\omega_{1} \omega^{\prime}+\omega_{2} i \omega^{\prime}, \quad \omega^{\prime \prime \prime}=\left(\omega_{1}^{2}-2 \omega_{2}+k R_{k} \omega_{2}\right) \omega^{\prime}+3 \omega_{1} \omega_{2} i \omega^{\prime} .
$$

Now we take any $\psi \in C^{2}\left(\mathbb{S}^{1}, \mathbb{R}\right)$ and we look for an explicit formula for $\mathcal{J}\left(\psi \omega^{\prime}\right)$. Clearly $L^{\prime}(\omega)\left(\psi \omega^{\prime}\right)=0$, as $\omega^{\prime} \cdot i \omega^{\prime} \equiv 0$. Direct computations based on (3.16) give

$$
\begin{aligned}
&-\left(\psi \omega^{\prime}\right)^{\prime \prime}+k R_{k} i\left(\psi \omega^{\prime}\right)^{\prime}=\left(-\psi^{\prime \prime}-2 \omega_{1} \psi^{\prime}-\left(\omega_{1}^{2}-2 \omega_{2}^{2}+2 k R_{k} \omega_{2}\right) \psi\right) \omega^{\prime} \\
&+\left(\left(k R_{k}-2 \omega_{2}\right) \psi^{\prime}+\left(k R_{k}-2 \omega_{2}\right) \omega_{1} \psi\right) i \omega^{\prime} \\
& \\
& A_{1}\left(\psi \omega^{\prime}\right)=2 \omega_{1} \psi^{\prime}-\left(2 \omega_{2}^{2}-2 k R_{k} \omega_{2}-\omega_{1}^{2}\right) \psi \\
& A_{2}\left(\psi \omega^{\prime}\right)=2\left(k R_{k}-\omega_{2}\right) \psi^{\prime}-\left(k R_{k}-3 \omega_{2}\right) \psi,
\end{aligned}
$$


and we find the formula

$$
\mathcal{J}\left(\psi \omega^{\prime}\right)=-\psi^{\prime \prime} \omega^{\prime}-k R_{k} \psi^{\prime} i \omega^{\prime}
$$

Now we handle $\mathcal{J}\left(\psi i \omega^{\prime}\right)$. From (3.5) we get

$$
k L^{\prime}(\omega)\left(\psi i \omega^{\prime}\right)=-k^{2} f_{\mathbb{S}^{1}} \omega_{2}^{-2}\left|\omega^{\prime}\right|^{2} \psi d x=-k^{2} R_{k}^{2} f_{\mathbb{S}^{1}} \psi d x .
$$

Then we use (3.2 3.5) and (3.16) to compute

$$
\begin{aligned}
-\left(\psi i \omega^{\prime}\right)^{\prime \prime}+k R_{k} i\left(\psi i \omega^{\prime}\right)^{\prime}= & \left(\left(2 \omega_{2}-k R_{k}\right) \psi^{\prime}+\left(3 \omega_{2}-k R_{k}\right) \omega_{1} \psi\right) \omega^{\prime} \\
& +\left(-\psi^{\prime \prime}-2 \omega_{1} \psi^{\prime}-\left(\omega_{1}^{2}-2 \omega_{2}^{2}+2 k R_{k} \omega_{2}\right) \psi\right) i \omega^{\prime} \\
A_{1}\left(\psi i \omega^{\prime}\right)= & -2\left(\omega_{2}-k R_{k}\right) \psi^{\prime}-\left(3 \omega_{2}-k R_{k}\right) \omega_{1} \psi \\
A_{2}\left(\psi i \omega^{\prime}\right)= & -2 \omega_{1} \psi^{\prime}+\left(\omega_{2}^{2}-k^{2} R_{k}^{2}-2 \omega_{1}^{2}\right) \psi .
\end{aligned}
$$

Since $R_{k}^{2}=\left|\omega-k R_{k} e_{2}\right|^{2}=|\omega|^{2}-2 k R_{k} \omega_{2}+k^{2} R_{k}^{2}$ by (3.2), we arrive at

$$
\mathcal{J}\left(\psi i \omega^{\prime}\right)=k R_{k} \psi^{\prime} \omega^{\prime}+\left(-\psi^{\prime \prime}+R_{k}^{2} \psi-k^{2} R_{k}^{2} f_{\mathbb{S}^{1}} \psi d x\right) i \omega^{\prime},
$$

that together with (3.17) gives

$$
\begin{aligned}
\mathcal{J}\left(g_{1} \omega^{\prime}+g_{2} i \omega^{\prime}\right)=( & \left.-g_{1}^{\prime \prime}-k R_{k} g_{2}^{\prime}\right) \omega^{\prime} \\
& +\left(-g_{2}^{\prime \prime}+k R_{k} g_{1}^{\prime}+R_{k}^{2} g_{2}-k^{2} R_{k}^{2} f_{\mathbb{S}^{1}} g_{2} d x\right) i \omega^{\prime}
\end{aligned}
$$

and concludes the proof of (3.15). The proof of $i$ ) is complete; the formula in $i i$ ) is immediate, because $\omega^{\prime} \cdot i \omega^{\prime} \equiv 0$ and $\left|\omega^{\prime}\right|=R_{k} \omega_{2}$.

We are in position to prove the main result of this section.

Lemma 3.3 (Nondegeneracy) Let $z$ be any point in $\mathbb{H}^{2}$. The following facts hold.

i) $\operatorname{ker} J^{\prime}\left(\omega_{z}\right)=T_{\omega} \mathcal{S}$;

ii) If $J_{0}^{\prime}\left(\omega_{z}\right) \varphi \in T_{\omega} \mathcal{S}$, then $\varphi \in T_{\omega} \mathcal{S}$;

iii) For any $u \in T_{\omega} \mathcal{S}^{\perp}$ there exists a unique $\varphi \in C^{2}\left(\mathbb{S}^{1}, \mathbb{R}^{2}\right) \cap T_{\omega} \mathcal{S}^{\perp}$ such that $J_{0}^{\prime}\left(\omega_{z}\right) \varphi=u$.

Proof. We start by studying the kernel of the operator $B$ in (3.14). In coordinates, the linear problem $B g=0$ becomes

$$
-g_{1}^{\prime \prime}+k R_{k} g_{2}^{\prime}=0, \quad-g_{2}^{\prime \prime}-k R_{k} g_{1}^{\prime}+R_{k}^{2}\left(g_{2}-k^{2} f_{\mathbb{S}^{1}} g_{2} d x\right)=0,
$$


that is clearly equivalent to

$$
f_{\mathbb{S}^{1}} g_{2} d x=0, \quad-g_{1}^{\prime \prime}+k R_{k} g_{2}^{\prime}=0, \quad-g_{2}^{\prime \prime}-k R_{k} g_{1}^{\prime}+R_{k}^{2} g_{2}=0
$$

because $k>1$. The system (3.18) can be studied via elementary techniques. The conclusion is that $\operatorname{ker} B=\left\langle e_{1}, \gamma, \gamma^{\prime}\right\rangle$, where $\gamma=\frac{1}{R_{k}}\left(k R_{k} x_{1},-x_{2}\right)$. Since $\Phi\left(e_{1}\right)=\omega^{\prime}, \Phi(\gamma)=\omega$ and $\Phi\left(\gamma^{\prime}\right)=e_{1}-\omega^{\prime}$, thanks to Lemma 3.2 we have

$$
\operatorname{ker} J_{0}^{\prime}\left(\omega_{z}\right)=\Phi(\operatorname{ker} B)=\Phi\left(\left\langle e_{1}, \gamma, \gamma^{\prime}\right\rangle\right)=T_{\omega} \mathcal{S},
$$

and the first claim is proved.

Now we prove $i i)$. If $\tau:=J_{0}^{\prime}\left(\omega_{z}\right) \varphi \in T_{\omega} \mathcal{S}=\operatorname{ker} J^{\prime}\left(\omega_{z}\right)$, then $J_{0}^{\prime}\left(\omega_{z}\right) \tau=0$. Taking (3.13) into account, we obtain

$$
f_{\mathbb{S}^{1}}\left|J_{0}^{\prime}\left(\omega_{z}\right) \varphi\right|^{2} d x=f_{\mathbb{S}^{1}} J_{0}^{\prime}\left(\omega_{z}\right) \varphi \cdot \tau d x=f_{\mathbb{S}^{1}} J_{0}^{\prime}\left(\omega_{z}\right) \tau \cdot \varphi d x=0 .
$$

Thus $J_{0}^{\prime}\left(\omega_{z}\right) \varphi=0$, that means $\varphi \in T_{\omega} \mathcal{S}$.

It remains to prove $i i i)$. If $u \in T_{\omega} \mathcal{S}^{\perp}$, then $\Phi^{-1}\left(\omega_{2}^{2} u\right)$ is orthogonal to $\operatorname{ker} B$ by $\left.i i\right)$ in Lemma 3.2. One can compute the Fourier coefficients of the unique solution $g_{u} \in \operatorname{ker} B^{\perp}$ of the system $B g_{u}=\Phi^{-1}\left(\omega_{2}^{2} u\right)$. Then $J_{0}^{\prime}(\omega)\left(z_{2}^{2} \Phi\left(g_{u}\right)\right)=u$ by $\left.i\right)$ in Lemma 3.2. The function $\varphi$ defined as the $L^{2}$-projection of $z_{2}^{2} \Phi\left(g_{u}\right)$ on $T_{\omega} \mathcal{S}^{\perp}$ solves $J_{0}^{\prime}(\omega) \varphi=u$ as well, and is uniquely determined by $u$.

The lemma is completely proved.

\section{The perturbed problem}

Let $k>1, K \in C^{1}\left(\mathbb{H}^{2}\right)$ be given, and let $\varepsilon \in \mathbb{R}$ be a varying parameter. In this section we study the system

$$
u^{\prime \prime}-u_{2}^{-1} \Gamma\left(u^{\prime}\right)=L(u)(k+\varepsilon K(u)) i u^{\prime} . \quad\left(\mathcal{P}_{k+\varepsilon K}\right)
$$

We start with a necessary condition for the existence of solutions to $\mathcal{P}_{k+\varepsilon K}$ having some prescribed behavior as $\varepsilon \rightarrow 0$.

Theorem 4.1 Let $k>1, K \in C^{1}\left(\mathbb{H}^{2}\right)$, and $\varepsilon_{h} \rightarrow 0$ be given. For any integer $h$, let $u_{h} \in C^{2}\left(\mathbb{S}^{1}, \mathbb{H}^{2}\right) \backslash \mathbb{H}^{2}$ be a solution to

$$
u_{h}^{\prime \prime}=\left(u_{h}\right)_{2}^{-1} \Gamma\left(u_{h}^{\prime}\right)+L\left(u_{h}\right)\left(k+\varepsilon_{h} K\left(u_{h}\right)\right) i u_{h}^{\prime},
$$

and assume that

$$
L\left(u_{h}\right) \rightarrow L_{\infty}>0, \quad u_{h} \rightarrow U \text { uniformly, for some } U \in C^{0}\left(\mathbb{S}^{1}, \mathbb{H}^{2}\right) .
$$

Then there exist $\mu \in \mathbb{N}, \xi \in \mathbb{S}^{1}$ and a critical point $z \in \mathbb{H}^{2}$ for $F_{k}^{K}$, such that $U(x)=$ $\omega_{z}\left(\xi x^{\mu}\right)$. 
Proof. We have $\left|u_{h}^{\prime}\right| \equiv L\left(u_{h}\right)\left(u_{h}\right)_{2}$, thus the sequence $\left|u_{h}^{\prime}\right|$ is uniformly bounded. It follows that $u_{h}^{\prime \prime}$ is uniformly bounded as well, because $u_{h}$ solves $\mathcal{P}_{\varepsilon_{h}}$. Thus, $u_{h}^{\prime}$ is bounded in $C^{0, s}$ for any $s \in(0,1)$ and using $\left[\mathcal{P}_{\varepsilon_{h}}\right.$ again we infer that the sequence $u_{h}$ converges in $C^{2, s}$ for any $s \in(0,1)$. In particular, $U \in C^{2}\left(\mathbb{S}^{1}, \mathbb{H}^{2}\right), L_{\infty}=L(U)$ and $U$ solves

$$
U^{\prime \prime}=U_{2}^{-1} \Gamma\left(U^{\prime}\right)+L(U) k i U^{\prime}
$$

Lemma 3.1 applies and gives the existence of $\xi \in \mathbb{S}^{1}, z \in \mathbb{H}^{2}, \mu \in \mathbb{N}$ such that $U(x)=$ $\omega_{z}\left(\xi x^{\mu}\right)$ and $L_{\infty}=L(U)=\mu L(\omega)$.

It remains to prove that $z$ is a critical point for $F_{k}^{K}$. We rewrite $\left[\mathcal{P}_{\varepsilon_{h}}\right.$ in the form

$$
J_{0}\left(u_{h}\right)+\varepsilon_{h} L\left(u_{h}\right)\left(u_{h}\right)_{2}^{-2} K\left(u_{h}\right) i u_{h}^{\prime}=0,
$$

see (3.10). Then we test (4.1) with the functions $e_{1}$ and $u_{h}$. Taking (3.12) into account, we find

$$
f_{\mathbb{S}^{1}}\left(u_{h}\right)_{2}^{-2} K\left(u_{h}\right) e_{1} \cdot i u_{h}^{\prime} d x=0, f_{\mathbb{S}^{1}}\left(u_{h}\right)_{2}^{-2} K\left(u_{h}\right) u_{h} \cdot i u_{h}^{\prime} d x=0 .
$$

Since $u_{h} \rightarrow U(x)=\omega_{z}\left(\xi x^{\mu}\right)$, in the limit as $h \rightarrow \infty$ we obtain

$$
\mu f_{\mathbb{S}^{1}}\left(\omega_{z}\right)_{2}^{-2} K\left(\omega_{z}\right) e_{1} \cdot i \omega_{z}^{\prime} d x=0, \quad \mu f_{\mathbb{S}^{1}}\left(\omega_{z}\right)_{2}^{-2} K\left(\omega_{z}\right) \omega_{z} \cdot i \omega_{z}^{\prime} d x=0,
$$

that is,

$$
\partial_{z_{1}} A_{K}\left(\omega_{z}\right)=A_{K}^{\prime}\left(\omega_{z}\right) e_{1}=0, \quad \partial_{z_{2}} A_{K}\left(\omega_{z}\right)=A_{K}^{\prime}\left(\omega_{z}\right) \omega=0 .
$$

Thus $z$ is a critical point for $F_{k}^{K}$ because of (3.6).

\subsection{Finite dimensional reduction}

By Lemma 2.1, $k+\varepsilon K$-loops are the critical points of the functional

$$
E_{k+\varepsilon K}(u)=E_{k}(u)+\varepsilon A_{K}(u)=L(u)+k A_{1}(u)+\varepsilon A_{K}(u), \quad u \in C^{2}\left(\mathbb{S}^{1}, \mathbb{H}^{2}\right) \backslash \mathbb{H}^{2} .
$$

We introduce the $C^{1}$ function $J_{\varepsilon}: C^{2}\left(\mathbb{R}, \mathbb{H}^{2}\right) \backslash \mathbb{H}^{2} \rightarrow C^{0}\left(\mathbb{R}, \mathbb{H}^{2}\right)$,

$$
\begin{aligned}
J_{\varepsilon}(u) & =J_{0}(u)+\varepsilon L(u) u_{2}^{-2} K(u) i u^{\prime} \\
& =u_{2}^{-2}\left(-u^{\prime \prime}+u_{2}^{-1} \Gamma\left(u^{\prime}\right)+L(u)(k+\varepsilon K(u)) i u^{\prime}\right),
\end{aligned}
$$

compare with (3.10), so that

$$
L(u) E_{k+\varepsilon K}^{\prime}(u) \varphi=f_{\mathbb{S}^{1}} J_{\varepsilon}(u) \cdot \varphi d x, \quad u \in C^{2}\left(\mathbb{S}^{1}, \mathbb{H}^{2}\right), \varphi \in C^{2}\left(\mathbb{S}^{1}, \mathbb{R}^{2}\right) .
$$

We will look for critical points for $E_{k+\varepsilon K}$ by solving the problem $J_{\varepsilon}(u)=0$. 
First, we notice that $E_{k+\varepsilon K}(u \circ \xi)=E_{k+\varepsilon K}(u)$ for any $\xi \in \mathbb{S}^{1}$, that implies

$$
f_{\mathbb{S}^{1}} J_{\varepsilon}(u) \cdot u^{\prime} d x=0 \quad \text { for any } \varepsilon \in \mathbb{R}, u \in C^{2}\left(\mathbb{S}^{1}, \mathbb{H}^{2}\right) \backslash \mathbb{H}^{2} .
$$

In the next crucial lemma we carry out the Lyapunov-Schmidt procedure, in which we take advantage of the variational structure of problem $\left(\overline{\mathcal{P}_{k+\varepsilon K}}\right)$.

Lemma 4.2 Let $\Omega \Subset \mathbb{H}^{2}$ be a given open set. There exist $\bar{\varepsilon}>0$ and a $C^{1}$ function

$$
[-\bar{\varepsilon}, \bar{\varepsilon}] \times \bar{\Omega} \rightarrow C^{2}\left(\mathbb{S}^{1}, \mathbb{H}^{2}\right) \backslash \mathbb{H}^{2}, \quad(\varepsilon, z) \mapsto u_{z}^{\varepsilon}
$$

such that the following facts hold.

i) $u_{z}^{\varepsilon}$ is an embedded loop and $u_{z}^{0}=\omega_{z}$;

ii) $u_{z}^{\varepsilon}-\omega_{z} \in T_{\omega} \mathcal{S}^{\perp}$

iii) $J_{\varepsilon}\left(u_{z}^{\varepsilon}\right) \in T_{\omega} \mathcal{S}$. More precisely,

$$
\frac{1}{L\left(u_{z}^{\varepsilon}\right)} J_{\varepsilon}\left(u_{z}^{\varepsilon}\right)=\partial_{z_{1}}\left(E_{k+\varepsilon K}\left(u_{z}^{\varepsilon}\right)\right) e_{1}+\left(f_{\mathbb{S}^{1}}|\omega|^{2} d x\right)^{-1} \partial_{z_{2}}\left(E_{k+\varepsilon K}\left(u_{z}^{\varepsilon}\right)\right) \omega
$$

iv) $A s \varepsilon \rightarrow 0$, we have

$$
E_{k+\varepsilon K}\left(u_{z}^{\varepsilon}\right)-E_{k+\varepsilon K}\left(\omega_{z}\right)=o(\varepsilon)
$$

uniformly on $\Omega$, together with the derivatives with respect to the variable $z$.

Proof. In order to shorten formulae, for $r>0, m \in\{0,2\}$ and $\delta>0$ we write

$$
\begin{gathered}
\Omega_{r}=\left\{z \in \mathbb{R}^{2} \mid \operatorname{dist}(z, \Omega)<r\right\}, \\
C^{m}=C^{m}\left(\mathbb{S}^{1}, \mathbb{R}^{2}\right), \quad \mathcal{U}_{\delta}:=\left\{\eta \in C^{2}|| \eta(x) \mid<\delta \text { for any } x \in \mathbb{S}^{1}\right\} .
\end{gathered}
$$

Take $r, \delta>0$ small enough, so that $\bar{\Omega}_{2 r} \subset \mathbb{H}^{2}$ and $\omega_{z}+\eta \in C^{2}\left(\mathbb{S}^{1}, \mathbb{H}^{2}\right) \backslash \mathbb{H}^{2}$ for any $z \in \bar{\Omega}_{2 r}, \eta \in \mathcal{U}_{\delta}$. Consider the differentiable function

$$
\mathcal{F}:\left(\mathbb{R} \times \Omega_{2 r}\right) \times \mathcal{U}_{\delta} \times\left(\mathbb{R} \times \mathbb{R}^{2}\right) \rightarrow C^{0} \times\left(\mathbb{R} \times \mathbb{R}^{2}\right), \quad \mathcal{F}=\left(\mathcal{F}_{1}, \mathcal{F}_{2}\right),
$$

whose coordinates

$$
\mathcal{F}_{1}:\left(\mathbb{R} \times \Omega_{2 r}\right) \times \mathcal{U}_{\delta} \times\left(\mathbb{R} \times \mathbb{R}^{2}\right) \rightarrow C^{0}, \quad \mathcal{F}_{2}:\left(\mathbb{R} \times \Omega_{2 r}\right) \times \mathcal{U}_{\delta} \times\left(\mathbb{R} \times \mathbb{R}^{2}\right) \rightarrow \mathbb{R} \times \mathbb{R}^{2}
$$

are given by

$$
\begin{aligned}
\mathcal{F}_{1}(\varepsilon, z ; \eta ; t, \vartheta) & =J_{\varepsilon}\left(\omega_{z}+\eta\right)-t \omega^{\prime}-\vartheta_{1} e_{1}-\vartheta_{2} \omega, \\
\mathcal{F}_{2}(\varepsilon, z ; \eta ; t, \vartheta) & =\left(f_{\mathbb{S}^{1}} \eta \cdot \omega^{\prime} d x, f_{\mathbb{S}^{1}} \eta_{1} d x, f_{\mathbb{S}^{1}} \eta \cdot \omega d x\right) .
\end{aligned}
$$


Take $z \in \Omega_{2 r}$ and notice that $\mathcal{F}(0, z ; 0 ; 0,0)=0$ because $J_{0}\left(\omega_{z}\right)=0$. The next goal is to solve the equation $\mathcal{F}(\varepsilon, z ; \eta ; t, \vartheta)=(0,0)$ in a neighborhood of $(\varepsilon, z)=(0, z),(\eta ; t, \vartheta)=$ $(0 ; 0,0)$ via the implicit function theorem. Let

$$
\mathcal{L}=\left(\mathcal{L}_{1}, \mathcal{L}_{2}\right): C^{2} \times\left(\mathbb{R} \times \mathbb{R}^{2}\right) \rightarrow C^{0} \times\left(\mathbb{R} \times \mathbb{R}^{2}\right)
$$

be the differential of $\mathcal{F}(0, z ; \cdot ; \cdot, \cdot)$ computed at $(\eta ; t, \vartheta)=(0 ; 0,0) \in C^{2} \times\left(\mathbb{R} \times \mathbb{R}^{2}\right)$. We need to prove that $\mathcal{L}$ is invertible. Explicitly, we have

$$
\begin{aligned}
& \mathcal{L}_{1}: C^{2} \times\left(\mathbb{R} \times \mathbb{R}^{2}\right) \rightarrow C^{0}, \quad \mathcal{L}_{1}(\varphi ; a, p)=J_{0}^{\prime}\left(\omega_{z}\right) \varphi-a \omega^{\prime}-p_{1} e_{1}-p_{2} \omega \\
& \mathcal{L}_{2}: C^{2} \times\left(\mathbb{R} \times \mathbb{R}^{2}\right) \rightarrow \mathbb{R} \times \mathbb{R}^{2}, \quad \mathcal{L}_{2}(\varphi ; a, p)=(\underbrace{}_{\mathbb{S}^{1}} \varphi \cdot \omega^{\prime} d x, f_{\mathbb{S}^{1}} \varphi_{1} d x, f_{\mathbb{S}^{1}} \varphi \cdot \omega d x) .
\end{aligned}
$$

If $\mathcal{L}_{1}(\varphi ; a, p)=0$ then $J_{0}^{\prime}\left(\omega_{z}\right) \varphi \in T_{\omega} \mathcal{S}$, hence $\varphi \in T_{\omega} \mathcal{S}$ by $\left.i i\right)$ in Lemma 3.3. If $\mathcal{L}_{2}(\varphi ; a, p)=$ 0 then $\varphi \in T_{\omega} \mathcal{S}^{\perp}$. Therefore, the operator $\mathcal{L}$ is injective.

To prove surjectivity take $u \in C^{0},(b, q) \in \mathbb{R} \times \mathbb{R}^{2}$. We have to find $\varphi \in C^{2},(a, p) \in$ $\mathbb{R} \times \mathbb{R}^{2}$ satisfying $\mathcal{L}_{1}(\varphi ; a, p)=u$ and $\mathcal{L}_{2}(\varphi ; a, p)=\left(b, q_{1}, q_{2}\right)$, that is,

$$
\begin{gathered}
J_{0}^{\prime}\left(\omega_{z}\right) \varphi=u+a \omega^{\prime}+p_{1} e_{1}+p_{2} \omega \\
f_{\mathbb{S}^{1}} \varphi \cdot \omega^{\prime} d x=b, \quad f_{\mathbb{S}^{1}} \varphi_{1} d x=q_{1}, \quad f_{\mathbb{S}^{1}} \varphi \cdot \omega d x=q_{2} .
\end{gathered}
$$

By (3.13), for any $\varphi \in C^{2}, \tau \in T_{\omega} \mathcal{S}=\left\langle\omega^{\prime}, e_{1}, \omega\right\rangle=\operatorname{ker} J_{0}^{\prime}\left(\omega_{z}\right)$ we have

$$
f_{\mathbb{S}^{1}} J_{0}^{\prime}\left(\omega_{z}\right) \varphi \cdot \tau d x=f_{\mathbb{S}^{1}} J_{0}^{\prime}\left(\omega_{z}\right) \tau \cdot \varphi d x=0
$$

Thus the unknowns $a \in \mathbb{R}$ and $p=\left(p_{1}, p_{2}\right) \in \mathbb{R}^{2}$ are determined by the condition

$$
f_{\mathbb{S}^{1}} u \cdot \tau d x+a f f_{\mathbb{S}^{1}} \omega^{\prime} \cdot \tau d x+p_{1} f_{\mathbb{S}^{1}} e_{1} \cdot \tau d x+p_{2} f_{\mathbb{S}^{1}} \omega \cdot \tau d x=0 \quad \text { for any } \tau \in T_{\omega} \mathcal{S} .
$$

Now we look for the $L^{2}$ projection of the unknown function $\varphi$ on $T_{\omega} \mathcal{S}$ and its $L^{2}$ projection on $T_{\omega} \mathcal{S}^{\perp}$. The tangential component $\varphi^{\top} \in T_{\omega} \mathcal{S}=\left\langle\omega^{\prime}, e_{1}, \omega\right\rangle$ is uniquely determined by (4.7). Next, we notice that $u+a \omega^{\prime}+p_{1} e_{1}+p_{2} \omega \in T_{\omega} \mathcal{S}^{\perp}$ by (4.8); then we use iii) in Lemma 3.3 to find $\varphi^{\perp} \in C^{2} \cap T_{\omega} \mathcal{S}^{\perp}$ such that

$$
J_{0}^{\prime}\left(\omega_{z}\right) \varphi^{\perp}=u+a \omega^{\prime}+p_{1} e_{1}+p_{2} \omega
$$

The function $\varphi=\varphi^{\top}+\varphi^{\perp}$ solves (4.6) because $J_{0}^{\prime}\left(\omega_{z}\right) \varphi=J_{0}^{\prime}\left(\omega_{z}\right) \varphi^{\perp}$, and surjectivity is proved. 
We can now apply the implicit function theorem for any fixed $z \in \Omega_{2 r}$. Actually, thanks a compactness argument we have that there exist $\varepsilon^{\prime}>0$ and (uniquely determined) $C^{1}$ functions

$$
\begin{array}{lll}
\eta:\left(-\varepsilon^{\prime}, \varepsilon^{\prime}\right) \times \Omega_{r} \rightarrow \mathcal{U}_{\delta} \subset C^{2} & t:\left(-\varepsilon^{\prime}, \varepsilon^{\prime}\right) \times \Omega_{r} \rightarrow \mathbb{R} & \vartheta:\left(-\varepsilon^{\prime}, \varepsilon^{\prime}\right) \times \Omega_{r} \rightarrow \mathbb{R}^{2} \\
\eta:(\varepsilon, z) \mapsto \eta^{\varepsilon}(z) & t:(\varepsilon, z) \mapsto t^{\varepsilon}(z), & \vartheta:(\varepsilon, z) \mapsto \vartheta^{\varepsilon}(z)
\end{array}
$$

such that

$$
\eta^{0}(z)=0, \quad t^{0}(z)=0, \quad \vartheta^{0}(z)=0, \quad \mathcal{F}\left(\varepsilon, z ; \eta^{\varepsilon}(z) ; t^{\varepsilon}(z), \vartheta^{\varepsilon}(z)\right)=0
$$

We introduce the $C^{1}$ function

$$
\left(-\varepsilon^{\prime}, \varepsilon^{\prime}\right) \times \Omega_{r} \rightarrow C^{2}\left(\mathbb{S}^{1}, \mathbb{H}^{2}\right) \backslash \mathbb{H}^{2}, \quad(\varepsilon, z) \mapsto u_{z}^{\varepsilon}:=\omega_{z}+\eta^{\varepsilon}(z),
$$

that clearly satisfies $u_{z}^{0}=\omega_{z}$. Since $\omega_{z}$ is embedded, then $u_{z}^{\varepsilon}$ is embedded as well, provided that $\varepsilon^{\prime}$ is small enough. Moreover we have

$$
\begin{gathered}
J_{\varepsilon}\left(u_{z}^{\varepsilon}\right)=t^{\varepsilon}(z) \omega^{\prime}+\vartheta_{1}^{\varepsilon}(z) e_{1}+\vartheta_{2}^{\varepsilon}(z) \omega \in T_{\omega} \mathcal{S} \\
f_{\mathbb{S}^{1}}\left(u_{z}^{\varepsilon}-\omega_{z}\right) \cdot \omega^{\prime} d x=f_{\mathbb{S}^{1}}\left(u_{z}^{\varepsilon}-\omega_{z}\right) \cdot e_{1} d x=f_{\mathbb{S}^{1}}\left(u_{z}^{\varepsilon}-\omega_{z}\right) \cdot \omega d x=0,
\end{gathered}
$$

and (4.10) shows that ii) is fulfilled.

Since integration by parts gives

$$
f_{\mathbb{S}^{1}} \omega_{z} \cdot \omega^{\prime} d x=0, \quad f_{\mathbb{S}^{1}} \omega_{z} \cdot e_{1} d x=z_{1}, \quad f_{\mathbb{S}^{1}} \omega_{z} \cdot \omega d x=z_{2} \underset{\mathbb{S}^{1}}{f}|\omega|^{2} d x,
$$

we can rewrite the orthogonality conditions (4.10) in the following, equivalent way:

$$
\underset{\mathbb{S}^{1}}{f} u_{z}^{\varepsilon} \cdot \omega^{\prime} d x=0, \quad f_{\mathbb{S}^{1}} u_{z}^{\varepsilon} \cdot e_{1} d x=z_{1}, \quad f_{\mathbb{S}^{1}} u_{z}^{\varepsilon} \cdot \omega d x=z_{2} f_{\mathbb{S}^{1}}|\omega|^{2} d x .
$$

Our next aim is to show that $t^{\varepsilon}(z)=0$ for any $z \in \bar{\Omega}$, provided that $\varepsilon$ is small enough. We have that $\left\|\left(u_{z}^{\varepsilon}\right)^{\prime}-\omega_{z}^{\prime}\right\|_{\infty}=o(1)$ as $\varepsilon \rightarrow 0$, uniformly for $z \in \bar{\Omega}$. Thus

$$
f_{\mathbb{S}^{1}}\left(u_{z}^{\varepsilon}\right)^{\prime} \cdot \omega^{\prime} d x=f_{\mathbb{S}^{1}} \omega_{z}^{\prime} \cdot \omega^{\prime} d x+o(1)=z_{2} f_{\mathbb{S}^{1}}\left|\omega^{\prime}\right|^{2} d x+o(1) .
$$

In particular, there exists $\bar{\varepsilon} \in\left(0, \varepsilon^{\prime}\right)$ such that $\int_{\mathbb{S}^{1}}\left(u_{z}^{\varepsilon}\right)^{\prime} \cdot \omega^{\prime} d x$ is bounded away from 0 if $(\varepsilon, z) \in[-\bar{\varepsilon}, \bar{\varepsilon}] \times \bar{\Omega}$. On the other hand, using (4.3), (4.9), integration by parts and (4.11), 
we have

$$
\begin{aligned}
0 & =f_{\mathbb{S}^{1}} J_{\varepsilon}\left(u_{z}^{\varepsilon}\right) \cdot\left(u_{z}^{\varepsilon}\right)^{\prime} d x \\
& =t^{\varepsilon}(z) f_{\mathbb{S}^{1}}\left(u_{z}^{\varepsilon}\right)^{\prime} \cdot \omega^{\prime} d x+\vartheta_{1}^{\varepsilon}(z) f_{\mathbb{S}^{1}}\left(u_{z}^{\varepsilon}\right)^{\prime} \cdot e_{1} d x+\vartheta_{2}^{\varepsilon}(z) f_{\mathbb{S}^{1}}\left(u_{z}^{\varepsilon}\right)^{\prime} \cdot \omega d x \\
& =t^{\varepsilon}(z) f_{\mathbb{S}^{1}}\left(u_{z}^{\varepsilon}\right)^{\prime} \cdot \omega^{\prime} d x-\vartheta_{2}^{\varepsilon}(z) f_{\mathbb{S}^{1}} u_{z}^{\varepsilon} \cdot \omega^{\prime} d x=t^{\varepsilon}(z) f_{\mathbb{S}^{1}}\left(u_{z}^{\varepsilon}\right)^{\prime} \cdot \omega^{\prime} d x .
\end{aligned}
$$

We see that $t^{\varepsilon}(z)=0$ for any $(\varepsilon, z) \in[-\bar{\varepsilon}, \bar{\varepsilon}] \times \bar{\Omega}$, and therefore

$$
J_{\varepsilon}\left(u_{z}^{\varepsilon}\right)=\vartheta_{1}^{\varepsilon}(z) e_{1}+\vartheta_{2}^{\varepsilon}(z) \omega .
$$

Now we compute the derivatives of the function $z \mapsto E_{k+\varepsilon K}\left(u_{z}^{\varepsilon}\right)$ via (4.2) and (4.12). For $j=1,2$ we obtain

$$
\begin{aligned}
L\left(u_{z}^{\varepsilon}\right) \partial_{z_{j}}\left(E_{k+\varepsilon K}\left(u_{z}^{\varepsilon}\right)\right) & =L\left(u_{z}^{\varepsilon}\right) E_{k+\varepsilon K}^{\prime}\left(u_{z}^{\varepsilon}\right) \partial_{z_{j}} u_{z}^{\varepsilon}=f_{\mathbb{S}^{1}} J_{\varepsilon}\left(u_{z}^{\varepsilon}\right) \cdot \partial_{z_{j}} u_{z}^{\varepsilon} d x \\
& =\vartheta_{1}^{\varepsilon}(z) f_{\mathbb{S}^{1}} \partial_{z_{j}} u_{z}^{\varepsilon} \cdot e_{1} d x+\vartheta_{2}^{\varepsilon}(z) f_{\mathbb{S}^{1}} \partial_{z_{j}} u_{z}^{\varepsilon} \cdot \omega d x \\
& =\vartheta_{1}^{\varepsilon}(z) \partial_{z_{j}}\left(f_{\mathbb{S}^{1}} u_{z}^{\varepsilon} \cdot e_{1} d x\right)+\vartheta_{2}^{\varepsilon}(z) \partial_{z_{j}}\left(f_{\mathbb{S}^{1}} u_{z}^{\varepsilon} \cdot \omega d x\right) .
\end{aligned}
$$

Then we use (4.11) to infer

$$
L\left(u_{z}^{\varepsilon}\right) \partial_{z_{1}}\left(E_{k+\varepsilon K}\left(u_{z}^{\varepsilon}\right)\right)=\vartheta_{1}^{\varepsilon}(z), \quad L\left(u_{z}^{\varepsilon}\right) \partial_{z_{2}}\left(E_{k+\varepsilon K}\left(u_{z}^{\varepsilon}\right)\right)=\vartheta_{2}^{\varepsilon}(z)\left(f_{\mathbb{S}^{1}}|\omega|^{2} d x\right),
$$

that compared with (4.12) give (4.4).

It remains to prove $i v)$. Take $z \in \bar{\Omega}$ and consider the function

$$
f_{z}(\varepsilon)=E_{k+\varepsilon K}\left(u_{z}^{\varepsilon}\right)=E_{k}\left(u_{z}^{\varepsilon}\right)+\varepsilon A_{K}\left(u_{z}^{\varepsilon}\right), \quad f_{z} \in C^{1}(-\bar{\varepsilon}, \bar{\varepsilon}) .
$$

Clearly $f_{z}(0)=E_{k}\left(\omega_{z}\right)$. To compute $f_{z}^{\prime}(0)$ notice that $\partial_{\varepsilon} u_{z}^{\varepsilon}$ remains bounded in $C^{2}(\bar{\Omega})$ as $\varepsilon \rightarrow 0$, because the function $(\varepsilon, z) \mapsto u_{z}^{\varepsilon}$ is of class $C^{1}$. Thus $A_{K}^{\prime}\left(u_{z}^{\varepsilon}\right)\left(\partial_{\varepsilon} u_{z}^{\varepsilon}\right)$ remains bounded as well. Further, $E_{k}^{\prime}\left(u_{z}^{\varepsilon}\right) \rightarrow E_{k}^{\prime}\left(\omega_{z}\right)=0$ in the norm operator because $u_{z}^{\varepsilon} \rightarrow \omega_{z}$ in $C^{2}$ and since $\omega_{z}$ is a $k$-loop. We infer that

$$
f_{z}^{\prime}(0)=E_{k}^{\prime}\left(\omega_{z}\right)\left(\partial_{\varepsilon} u_{z}^{\varepsilon}\right)+A_{K}\left(u_{z}^{\varepsilon}\right)+o(1)=A_{K}\left(\omega_{z}\right)+o(1)
$$

uniformly on $\bar{\Omega}$. In fact we proved that

$$
f_{z}(\varepsilon)=E_{k+\varepsilon K}\left(u_{z}^{\varepsilon}\right)=E_{k}\left(\omega_{z}\right)+\varepsilon A_{K}\left(u_{z}^{\varepsilon}\right)+o(1)
$$


uniformly on $\bar{\Omega}$ as $\varepsilon \rightarrow 0$. That is, (4.5) holds true "at the zero order".

To conclude the proof we have to handle $\partial_{z_{j}}\left(E_{k+\varepsilon K}\left(u_{z}^{\varepsilon}\right)-E_{k+\varepsilon K}\left(\omega_{z}\right)\right)$ for $j=1,2$. Since $J_{\varepsilon}(u)=J_{0}(u)+\varepsilon L(u) u_{2}^{-2} K(u) i u^{\prime}$, we can rewrite (4.4) as follows,

$$
\begin{aligned}
\partial_{z_{1}}\left(E_{k+\varepsilon K}\left(u_{z}^{\varepsilon}\right)\right) e_{1}+\left(f_{\mathbb{S}^{1}}|\omega|^{2} d x\right)^{-1} \partial_{z_{2}}\left(E_{k+\varepsilon K}\left(u_{z}^{\varepsilon}\right)\right) \omega \\
=\frac{1}{L\left(u_{z}^{\varepsilon}\right)} J_{0}\left(u_{z}^{\varepsilon}\right)+\varepsilon\left(u_{z}^{\varepsilon}\right)_{2}^{-2} K\left(u_{z}^{\varepsilon}\right) i\left(u_{z}^{\varepsilon}\right)^{\prime} .
\end{aligned}
$$

Recall that $J_{0}\left(u_{z}^{\varepsilon}\right)$ is orthogonal to $e_{1}$ in $L^{2}$, see the second identity in (3.12). We test (4.13) with $e_{1}$ to obtain

$$
\partial_{z_{1}}\left(E_{k+\varepsilon K}\left(u_{z}^{\varepsilon}\right)\right)=\varepsilon f_{\mathbb{S}^{1}}\left(u_{z}^{\varepsilon}\right)_{2}^{-2} K\left(u_{z}^{\varepsilon}\right) e_{1} \cdot i\left(u_{z}^{\varepsilon}\right)^{\prime} d x=\varepsilon A_{K}^{\prime}\left(u_{z}^{\varepsilon}\right) e_{1}
$$

by (2.5). Since $\partial_{z_{1}}\left(E_{k+\varepsilon K}\left(\omega_{z}\right)\right)=\partial_{z_{1}}\left(E_{k}(\omega)+\varepsilon A_{K}\left(\omega_{z}\right)\right)=\varepsilon A_{K}^{\prime}\left(\omega_{z}\right) e_{1}$, we get

$$
\partial_{z_{1}}\left(E_{k+\varepsilon K}\left(u_{z}^{\varepsilon}\right)-E_{k+\varepsilon K}\left(\omega_{z}\right)\right)=\varepsilon\left(A_{K}^{\prime}\left(u_{z}^{\varepsilon}\right) e_{1}-A_{K}^{\prime}\left(\omega_{z}\right) e_{1}\right)=o(\varepsilon)
$$

because of the continuity of $A_{K}^{\prime}(\cdot)$ and since $u_{z}^{\varepsilon} \rightarrow \omega_{z}$.

To handle the derivative with respect to $z_{2}$ we test (4.13) with $u_{z}^{\varepsilon}$. Since $J_{0}\left(u_{z}^{\varepsilon}\right)$ is orthogonal to $u_{z}^{\varepsilon}$ in $L^{2}$ by (3.12), using also (4.11) we obtain

$$
z_{1} \partial_{z_{1}}\left(E_{k+\varepsilon K}\left(u_{z}^{\varepsilon}\right)\right)+z_{2} \partial_{z_{2}}\left(E_{k+\varepsilon K}\left(u_{z}^{\varepsilon}\right)\right)=\varepsilon f_{\mathbb{S}^{1}}\left(u_{z}^{\varepsilon}\right)_{2}^{-2} K\left(u_{z}^{\varepsilon}\right) u_{z}^{\varepsilon} \cdot i\left(u_{z}^{\varepsilon}\right)^{\prime} d x=\varepsilon A_{K}^{\prime}\left(u_{z}^{\varepsilon}\right) u_{z}^{\varepsilon},
$$

that compared with (4.14) gives

$$
z_{2} \partial_{z_{2}}\left(E_{k+\varepsilon K}\left(u_{z}^{\varepsilon}\right)\right)=\varepsilon A_{K}^{\prime}\left(u_{z}^{\varepsilon}\right)\left(u_{z}^{\varepsilon}-z_{1} e_{1}\right) .
$$

From $z_{2} \partial_{z_{2}}\left(E_{k+\varepsilon K}\left(\omega_{z}\right)\right)=z_{2} \partial_{z_{2}}\left(E_{k}(\omega)+\varepsilon A_{K}\left(\omega_{z}\right)\right)=z_{2} \varepsilon A_{K}^{\prime}\left(\omega_{z}\right) \omega=\varepsilon A_{K}^{\prime}\left(\omega_{z}\right)\left(\omega_{z}-z_{1} e_{1}\right)$, we conclude that

$$
z_{2} \partial_{z_{2}}\left(E_{k+\varepsilon K}\left(u_{z}^{\varepsilon}\right)-E_{k+\varepsilon K}\left(\omega_{z}\right)\right)=\varepsilon\left(A_{K}^{\prime}\left(u_{z}^{\varepsilon}\right)\left(u_{z}^{\varepsilon}-z_{1} e_{1}\right)-A_{K}^{\prime}\left(\omega_{z}\right)\left(\omega_{z}-z_{1} e_{1}\right)\right)=o(\varepsilon) .
$$

The lemma is completely proved.

\subsection{Existence results}

Proof of Theorem 1.1. We are assuming that there exists $r>0$ such that any function

$G \in C^{1}(\bar{A})$ satisfying $\left\|G+F_{k}^{K}\right\|_{C^{1}(\bar{A})}<r$ has a critical point in $A$. We recall also formula (3.6), that in particular gives

$$
E_{k+\varepsilon K}\left(\omega_{z}\right)=E_{k}\left(\omega_{z}\right)+\varepsilon A_{K}\left(\omega_{z}\right)=E_{k}(\omega)-\frac{\varepsilon}{2 \pi} F_{k}^{K}(z) .
$$


Take an open set $\Omega \in \mathbb{H}^{2}$ such that $A \Subset \Omega \Subset \mathbb{H}^{2}$, and let $(\varepsilon, z) \mapsto u_{z}^{\varepsilon},(\varepsilon, z) \in[-\bar{\varepsilon}, \bar{\varepsilon}] \times \bar{\Omega}$ be the function given by Lemma 4.2 . For $\varepsilon \neq 0$ consider the function

$$
G^{\varepsilon}(z)=\frac{2 \pi}{\varepsilon}\left(E_{k+\varepsilon K}\left(u_{z}^{\varepsilon}\right)-E_{k}(\omega)\right)
$$

and use (4.15) together with $i v$ ) in Lemma 4.2 to get

$$
\left\|G^{\varepsilon}+F_{k}^{K}\right\|_{C^{1}(\bar{A})}=\frac{2 \pi}{|\varepsilon|}\left\|E_{k+\varepsilon K}\left(u_{z}^{\varepsilon}\right)-E_{k+\varepsilon K}\left(\omega_{z}\right)\right\|=o(1)
$$

as $\varepsilon \rightarrow 0$. We see that for $\varepsilon$ small enough the function $G^{\varepsilon}$ has a critical point $z^{\varepsilon} \in A$. Since the derivatives of the function $z \mapsto E_{k+\varepsilon K}\left(u_{z}^{\varepsilon}\right)$ vanish at $z=z^{\varepsilon}$, then $J_{\varepsilon}\left(u_{z^{\varepsilon}}^{\varepsilon}\right)=0$ by (4.4). That is, $u_{z^{\varepsilon}}^{\varepsilon}$ is and embedded $k+\varepsilon K$ loop.

The last conclusion in Theorem 1.1 follows via a simple compactness argument and thanks to Theorem 4.1 .

In the next result we apply Theorem 1.1 to obtain the existence of $k+\varepsilon K$-loops that shrink to a stable critical point for the curvature function $K$, as $k \rightarrow \infty$.

Theorem 4.3 Let $K \in C^{1}\left(\mathbb{H}^{2}\right)$. Assume that $K$ has a stable critical point in an open set $A \Subset \mathbb{H}^{2}$. There exists $k_{0}>1$ such that for any $k>k_{0}$ and for every $\varepsilon$ close enough to 0 , there exists an embedded $(k+\varepsilon K)$-loop.

Moreover, let $k_{h} \rightarrow \infty, \varepsilon_{h} \rightarrow 0$ be given sequences. There exist subsequences $k_{h_{j}}, \varepsilon_{h_{j}}$, a point $z_{\infty} \in \bar{A}$ that is critical for $K$, and an embedded $\left(k_{h_{j}}+\varepsilon_{h_{j}} K\right)$-loop $u^{j}$ such that $u^{j}$ converges in $C^{2}\left(\mathbb{S}^{1}, \mathbb{H}^{2}\right)$ to the constant curve $z_{\infty}$, as $h \rightarrow \infty$.

Proof. Recall that $R_{k}=\left(k^{2}-1\right)^{-1 / 2}$. In order to simplify notations we put

$$
z^{k}:=\left(z_{1}, k R_{k} z_{2}\right)=z+\left(k R_{k}-1\right) z_{2} e_{2} \quad \text { for } z=\left(z_{1}, z_{2}\right) \in \mathbb{H}^{2} .
$$

Since $D_{\rho_{k}}^{\mathbb{H}}(z)=D_{R_{k} z_{2}}\left(z^{k}\right)$ we have

$$
F_{k}^{K}(z)=\int_{D_{R_{k} z_{2}}\left(z^{k}\right)} p_{2}^{-2} K(p) d p=\int_{D_{R_{k}}(0)}\left(q_{2}+k R_{k}\right)^{-2} K\left(z_{2} q+z^{k}\right) d q .
$$

We put $\phi_{K}(q)=q_{2}^{-2} K(q)$ and rewrite (4.16) as follows:

$$
\frac{1}{\pi R_{k}^{2} z_{2}^{2}} F_{k}^{K}(z)=\underset{D_{R_{k}}(0)}{f} \phi_{K}\left(z_{2} q+z^{k}\right) d q .
$$

Trivially $k R_{k}=k / \sqrt{k^{2}-1} \rightarrow 1$ and $\left|z^{k}-z\right|=\left(k R_{k}-1\right) z_{2} \rightarrow 0$ uniformly on $\bar{A}$, as $k \rightarrow \infty$. Since $\phi_{K} \in C^{1}\left(\mathbb{H}^{2}\right)$, it is easy to show that

$$
\frac{1}{\pi R_{k}^{2} z_{2}^{2}} F_{k}^{K}(z) \rightarrow \phi_{K}(z)=\frac{1}{z_{2}^{2}} K(z)
$$

in $C^{1}(\bar{A})$. It follows that for $k$ large enough, $F_{k}^{K}$ has stable critical point in $A \Subset \mathbb{H}^{2}$. Theorem 1.1 applies and gives the conclusion of the proof. 


\section{A Loops in the Euclidean plane}

The argument we used to prove Theorem 1.1 applies also in the easier Euclidean case. It is well known that the only embedded loops in $\mathbb{R}^{2}$ having prescribed constant curvature $k>0$ are circles of radius $1 / k$. We take as a reference circle the loop

$$
\omega(x)=\frac{1}{k} x, \quad x \in \mathbb{S}^{1} \subset \mathbb{R}^{2},
$$

that solves

$$
u^{\prime \prime}=L(u) k i u^{\prime}, \quad \text { where } \quad L(u):=\left(f_{\mathbb{S}^{1}}\left|u^{\prime}\right|^{2} d x\right)^{\frac{1}{2}}
$$

(in fact, $L(\omega) k=1$ and $\omega^{\prime \prime}=-\omega=i \omega^{\prime}$ ).

Let $K \in C^{1}\left(\mathbb{R}^{2}\right)$ be given. If a nonconstant function $u \in C^{2}\left(\mathbb{S}^{1}, \mathbb{R}^{2}\right)$ solves

$$
u^{\prime \prime}=L(u)(k+\varepsilon K(u)) i u^{\prime}
$$

then $\left|u^{\prime}\right|=L(u)$ is constant, and $u$ parameterizes a loop in $\mathbb{R}^{2}$ having Euclidean curvature $k+\varepsilon K$ at each point. Further, problem (A.1) admits a variational structure, see [5], [18]. More precisely, its nonconstant solutions are critical points of the energy functional

$$
E_{k+\varepsilon K}(u)=\left(f_{\mathbb{S}^{1}}\left|u^{\prime}\right|^{2} d x\right)^{\frac{1}{2}}+\varepsilon f_{\mathbb{S}^{1}} Q(u) \cdot i u^{\prime}, \quad u \in C^{2}\left(\mathbb{S}^{1}, \mathbb{R}^{2}\right) \backslash \mathbb{R}^{2},
$$

where the vectorfield $Q \in C^{1}\left(\mathbb{R}^{2}, \mathbb{R}^{2}\right)$ satisfies $\operatorname{div} Q=K$.

Arguing as for Theorem 4.1 one can prove a necessary conditions for the existence of solutions to A.1 for $\varepsilon=\varepsilon_{h} \rightarrow 0$.

Theorem A.1 Let $u_{h}$ be $a\left(k+\varepsilon_{h} K\right)$-loop solving (A.1) for $\varepsilon=\varepsilon_{h}$, and assume that

$$
L\left(u_{h}\right) \rightarrow L_{\infty}>0, \quad u_{h} \rightarrow U \text { uniformly, for some } U \in C^{0}\left(\mathbb{S}^{1}, \mathbb{R}^{2}\right) .
$$

Then $U(x)=\omega\left(\xi x^{\mu}\right)+z$ for some $\mu \in \mathbb{N}, \xi \in \mathbb{S}^{1}$ and $z \in \mathbb{R}^{2}$, that is a critical point for the Melnikov function

$$
F_{k}^{K}(z)=\int_{D_{\frac{1}{k}}(z)} K(q) d q, \quad F_{k}^{K}: \mathbb{R}^{2} \rightarrow \mathbb{R}
$$

In the Euclidean case we have the following existence result.

Theorem A.2 Let $k>0$ and $K \in C^{1}\left(\mathbb{R}^{2}\right)$ be given. Assume that $F_{k}^{K}$ has a stable critical point in an open set $A \Subset \mathbb{R}^{2}$. Then for every $\varepsilon \in \mathbb{R}$ close enough to 0 , there exists an embedded $(k+\varepsilon K)$-loop $u^{\varepsilon}: \mathbb{S}^{1} \rightarrow \mathbb{R}^{2}$.

Moreover, any sequence $\varepsilon_{h} \rightarrow 0$ has a subsequence $\varepsilon_{h_{j}}$ such that $u^{\varepsilon_{h_{j}}} \rightarrow \omega_{z_{0}}$ in $C^{2}\left(\mathbb{S}^{1}, \mathbb{R}^{2}\right)$ as $j \rightarrow \infty$, where $z_{0} \in A$ is a critical point for $F_{k}^{K}$. 
Sketch of the proof. We introduce the 3-dimensional space of embedded solutions to the unperturbed problem, namely

$$
\mathcal{S}=\left\{\omega \circ \xi+z \mid \xi \in \mathbb{S}^{1}, \quad z \in \mathbb{R}^{2}\right\}
$$

and the functions $J_{\varepsilon}: C^{2}\left(\mathbb{R}, \mathbb{R}^{2}\right) \backslash \mathbb{R}^{2} \rightarrow C^{0}\left(\mathbb{R}, \mathbb{R}^{2}\right), \varepsilon \in \mathbb{R}$, given by

$$
J_{\varepsilon}(u)=-u^{\prime \prime}+L(u)(k+\varepsilon K(u)) i u^{\prime}=J_{0}(u)+L(u) K(u) i u^{\prime} .
$$

We have $\mathcal{S} \subset\left\{J_{0}=0\right\}$. Since $J_{0}^{\prime}(\omega+z) \varphi=-\varphi^{\prime \prime}+i \varphi^{\prime}-k^{2}\left(f_{\mathbb{S}^{1}} \varphi \cdot \omega d x\right) \omega$, it is quite easy to check that

$$
T_{\omega+z} \mathcal{S}=\left\langle\omega^{\prime}, e_{1}, e_{2}\right\rangle=\operatorname{ker} J_{0}^{\prime}(\omega+z),
$$

and that $J_{0}^{\prime}(\omega+z): T_{\omega+z} \mathcal{S}^{\perp} \rightarrow T_{\omega+z} \mathcal{S}^{\perp}$ is invertible. The remaining part of the proof runs with minor changes.

Theorem 4.3 has its Euclidean correspondent as well. We omit the proof of the next result.

Theorem A.3 Let $K \in C^{1}\left(\mathbb{R}^{2}\right)$. Assume that $K$ has a stable critical point in an open set $A \Subset \mathbb{R}^{2}$. Then there exists $k_{0}>1$ such that for any fixed $k>k_{0}$, and for every $\varepsilon$ close enough to 0 , there exists an embedded $(k+\varepsilon K)$-loop $u^{k, \varepsilon}: \mathbb{S}^{1} \rightarrow \mathbb{R}^{2}$.

Moreover, there exist sequences $k_{h} \rightarrow \infty, \varepsilon_{h} \rightarrow 0$ such that $u^{k_{h}, \varepsilon_{h_{j}}} \rightarrow \omega_{z_{0}}$ in $C^{2}\left(\mathbb{S}^{1}, \mathbb{R}^{2}\right)$ as $j \rightarrow \infty$, where $z_{0} \in A$ is a critical point for $K$.

\section{References}

[1] A. Ambrosetti and M. Badiale, Variational perturbative methods and bifurcation of bound states from the essential spectrum, Proc. Roy. Soc. Edinburgh Sect. A 128 (1998), no. 6, $1131-1161$.

[2] A. Ambrosetti, Y. Li and A. Malchiodi, On the Yamabe problem and the scalar curvature problems under boundary conditions, Math. Ann. 322 (2002), no. 4, 667-699.

[3] A. Ambrosetti and A. Malchiodi, Perturbation methods and semilinear elliptic problems on $\mathbb{R}^{n}$, Progress in Mathematics, 240, Birkhäuser Verlag, Basel, 2006.

[4] V.I. Arnol'd, The first steps of symplectic topology, Uspekhi Mat. Nauk 41 (1986), no. 6(252), $3-18,229$.

[5] F. Bethuel, P. Caldiroli and M. Guida, Parametric surfaces with prescribed mean curvature, Rend. Sem. Mat. Univ. Politec. Torino 60 (2002), no. 4, 175-231 (2003).

[6] P. Caldiroli, $H$-bubbles with prescribed large mean curvature, Manuscripta Math. 113 (2004), no. 1, 125-142. 
[7] P. Caldiroli and R. Musina, $H$-bubbles in a perturbative setting: the finite-dimensional reduction method, Duke Math. J. 122 (2004), no. 3, 457-484.

[8] P. Caldiroli and M. Musso, Embedded tori with prescribed mean curvature, preprint arXiv:1709.08495 (2017).

[9] G. Contreras, L. Macarini and G.P. Paternain, Periodic orbits for exact magnetic flows on surfaces, Int. Math. Res. Not. 2004, no. 8, 361-387.

[10] M.M. Fall, Embedded disc-type surfaces with large constant mean curvature and free boundaries, Commun. Contemp. Math. 14 (2012), no. 6, 1250037, 35 pp.

[11] V. Felli, A note on the existence of $H$-bubbles via perturbation methods, Rev. Mat. Iberoamericana 21 (2005), no. 1, 163-178.

[12] V.L. Ginzburg, New generalizations of Poincaré's geometric theorem, Funktsional. Anal. i Prilozhen. 21 (1987), no. 2, 16-22, 96.

[13] V.L. Ginzburg, On the existence and non-existence of closed trajectories for some Hamiltonian flows, Math. Z. 223 (1996), no. 3, 397-409.

[14] J. Jost, Riemannian geometry and geometric analysis, fifth edition, Universitext, SpringerVerlag, Berlin, 2008.

[15] R. López, Constant mean curvature surfaces with boundary, Springer Monographs in Mathematics, Springer, Heidelberg, 2013.

[16] A. Mondino, The conformal Willmore functional: a perturbative approach, J. Geom. Anal. 23 (2013), no. 2, 764-811.

[17] R. Musina, The role of the spectrum of the Laplace operator on $\mathbb{S}^{2}$ in the $H$-bubble problem, J. Anal. Math. 94 (2004), 265-291.

[18] R. Musina, Planar loops with prescribed curvature: existence, multiplicity and uniqueness results, Proc. Amer. Math. Soc. 139 (2011), no. 12, 4445-4459.

[19] S.P. Novikov and I.A. Taǐmanov, Periodic extremals of multivalued or not everywhere positive functionals, Dokl. Akad. Nauk SSSR 274 (1984), no. 1, 26-28.

[20] M. Schneider, Closed magnetic geodesics on $S^{2}$, J. Differential Geom. 87 (2011), no. 2, $343-388$.

[21] M. Schneider, Closed magnetic geodesics on closed hyperbolic Riemann surfaces, Proc. Lond. Math. Soc. (3) 105 (2012), no. 2, 424-446.

[22] F. Schlenk, Applications of Hofer's geometry to Hamiltonian dynamics, Comment. Math. Helv. 81 (2006), no. 1, 105-121.

[23] I.A. Taǐamanov, Closed extremals on two-dimensional manifolds, Russian Math. Surveys 47 (1992), no. 2, 163-211; translated from Uspekhi Mat. Nauk 47 (1992), no. 2(284), 143-185, 223.

[24] R. Ye, Foliation by constant mean curvature spheres, Pacific J. Math. 147 (1991), no. 2, 381-396. 